\title{
Never take candy from a stranger: the role of the bacterial glycome in host-pathogen interactions
}

\section{Christopher W Reid, Kelly M Fulton \& Susan M Twine ${ }^{\dagger}$}

†Author for correspondence: National Research Council - Institute for Biological Science, Ottawa, ON, KTA OR6, Canada, =1 Tel.. +1 6139497545 = Fax: +1 6139529092 = Susan.Twine@nrc-cnrc.gc.ca

With the comprehensive study and complete sequencing of the Haemophilus influenzae genome in 1995 came the term 'genomics' and the beginning of the 'omics' era. Since this time, several analogous fields, such as transcriptomics and proteomics, have emerged. While growth and advancement in these fields have increased understanding of microbial virulence, the study of bacterial glycomes is still in its infancy and little is known concerning their role in host-pathogen interactions. Bacterial glycomics is challenging owing to the diversity of glycoconjugate molecules, vast array of unusual sugars and limited number of analytical approaches available. However, recent advances in glycomics technologies offer the potential for exploration and characterization of both the structures and functions of components of bacterial glycomes in a systematic manner. Such characterization is a prerequisite for discerning the role of bacterial glycans in the interaction between host defences and bacterial virulence factors.

Carbohydrates constitute the most structurally diverse class of natural products and can serve many functions in cells and organisms [1]. In addition to their role in energy metabolism, carbohydrates are also found attached to proteins and lipids, or as loosely associated polysaccharides on the cell surface. Glycobiology encompasses the determination of the structure and function of complex carbohydrates (glycans). More recently, there has been a shift within glycobiology towards large-scale systematic studies of the entire complement of glycan structures within a given cell or organism, refered to as the 'glycome'. Glycomics, therefore, is the study of the significance of glycoconjugate assembly and expression in biological systems. Since glycosylation involves a series of metabolic events, some of the concepts in lipidomics [2] and metabolomics [3] are common to glycomics. As a field, glycomics has lagged behind genomics and proteomics, owing to the inherent difficulties in the isolation of glycans, the subsequent analysis of their structure and function and the limited number of different analytical techniques available for use in their study.

Surface polysaccharides represent the predominant structures on all bacterial cell surfaces, and they are often important players in the interactions between pathogens, their hosts and the environment. These structures can be involved in the maintenance of surface charge, phase variation and serum resistance. In bacteria, the role of these glycan moieties in symbiosis, pathogenesis, biofilm formation, cell-cell interactions and evasion of the immune response [4-7] is just beginning to be understood. In Gram-negative bacteria, the vast majority of carbohydrate moieties are found in lipopolysaccharides (LPS)/ lipooligosaccharides (LOS) or capsular polysaccharide (CPS). There are an increasing number of reports describing the modification of bacterial proteins with $\mathrm{N}$ - or $\mathrm{O}$-linked glycan moieties (Figure 1) (reviewed in [8,9]).

One of the main challenges in the study of glycomes is the complexity of carbohydrate structures. In mammals, carbohydrates are assembled from a group of approximately ten common monosaccharides. By contrast, the absolute number of potential monosaccharide building blocks in bacteria is unknown. Bacteria can synthesize pentose and heptose sugars not commonly found in mammals, and this diverse array of glycans is proving to be an analytical challenge. In addition, carbohydrate complexity increases as monosaccharides are assembled into linear and branched polymers based on the regiochemistry and stereochemistry $(\alpha$ or $\beta$ ) of the anomeric carbon in the glycosidic bond. For example, it is estimated that, for a hexasaccharide, all possible oligosaccharide isomers would yield approximately $10^{12}$ structures [10]. In addition to the monosaccharide and linkage diversity, a number of modifications to the monosaccharide subunits can occur, including methylation, sulfation, phosphorylation and, in some bacteria, the addition of amino acids $[11,12]$. Finally, the method of display of the glycan, by attachment to lipids or via $N$ - and $O$-linkage to proteins, adds further complexity to the glycome. This complexity is compounded by the

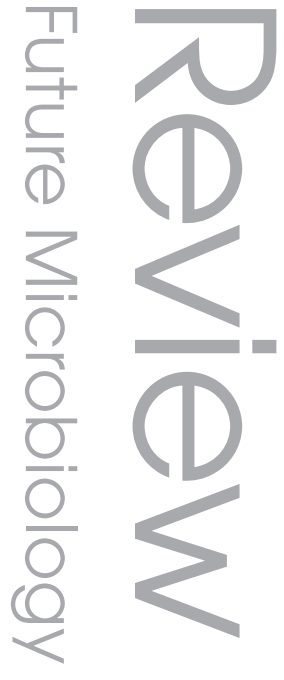

\section{Keywords}

- bacterial glycome

- Campylobacter jejuni

- capsular polysaccharide

- glycobiology = glycolipid

- glycomics

- glycoproteomics

- host-pathogen interactions

- lipopolysaccharide

- mass spectrometry $=$ NMR

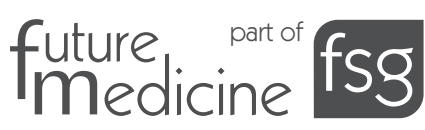




\section{Review Reid, Fulton \& Twine}

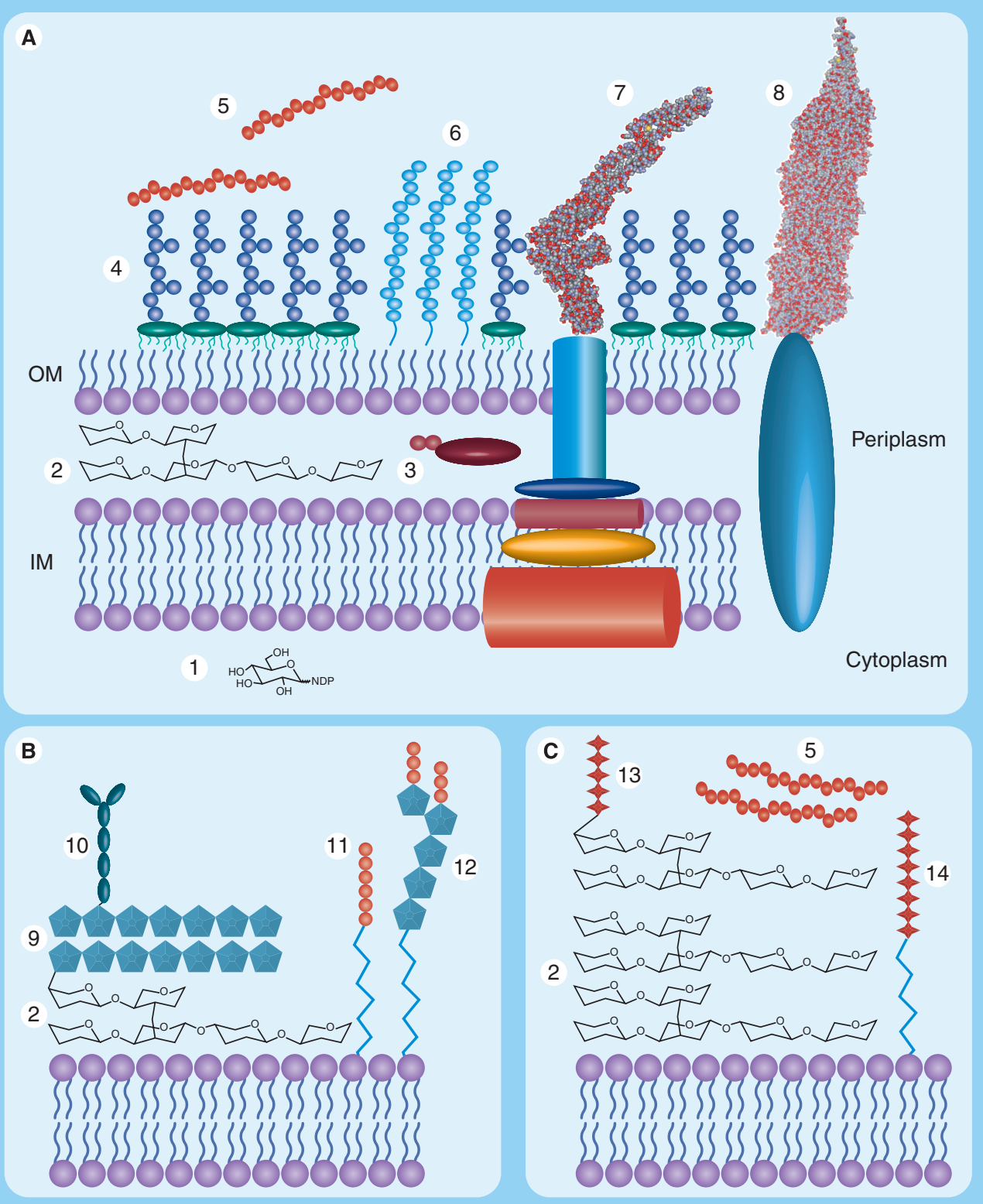

Figure 1. Microbial glycome diversity. (A) The Gram-negative cell showing the various components that constitute the glycome: (1) nucleotide-linked sugar precursors; (2) peptidoglycan; (3) N-linked glycoproteins; (4) lipopolysaccharide/oligosaccharide; (5) extracellular polysaccharide; (6) capsular polysaccharide; (7) O-linked protein glycosylation, FliC, Salmonella enterica (PDB: 1UCU); (8) pilin Neisseria gonorhoeae (PDB: 2HIL). (B) The mycobacterial glycome in addition to possessing nucleotide-linked sugar precursors and peptidoglycan the cell surface contains: (9) arabinogalactan, (10) mycolic acids, (11) lipomannan and (12) lipoarabinomannan. (C) The Gram-positive cell produces many of the same glycoconjugates as the Gram-negative cell, such as capsular polysaccharide, extracellular polysaccharide and a thicker peptidoglycan layer but, in addition, produces the cell surface components (13) teichoic and (14) lipoteichoic acids.

IM: Inner membrane; OM: Outer membrane.

nontemplate-driven nature of glycan biosynthesis. Taking all these factors into consideration, the analytical challenge to dissect these structures, and assign biological function, is substantial.

In this review we provide an overview of bacterial glycomes and the analytical techniques that are employed in their study. Owing to the diversity in this field, it is not feasible to discuss all the contributions made to bacterial glycomics; therefore, this review will focus on surveying the recent literature and describing select techniques that can be applied to the analysis of the bacterial glycome. We then focus upon how this work has specifically contributed to 
our understanding of the role of the bacterial glycome in the host-pathogen interactions of Campylobacter jejuni.

\section{Deciphering the bacterial glycome using mass spectrometry Mass spectrometry in bacterial glycoprotein discovery}

For many years, glycoproteins were thought to be expressed solely by eukaryotes and archaea, but it is now evident that glycoproteins are found in all domains of life. The first noneukaryotic glycoprotein was the surface layer protein (SLP) from the archaean Halobacterium salinarum [13,14]. More recently, research has demonstrated that several medically relevant human pathogens also modify their proteins with glycan moieties [15].

In the last decade, protein $N$-glycosylation (via asparagine residues) has been identified in the intestinal pathogen $C$. jejuni [16], and $O$-linked protein glycosylation (via serine or threonine residues) has been described in pathogens such as Helicobacter pylori [17], Pseudomonas aeruginosa [18,19], Neisseria spp. [20,21], Escherichia coli [22,23] and Clostridium difficile [24]. Protein glycosylation has been increasingly reported in both Gram-positive and Gram-negative bacteria, commonly including flagellins [9], pilins [25-27], fimbriae [28], cell surface proteins (e.g., adhesins [29-31]) and structural proteins (e.g., SLPs of Gram-positive bacteria [32]). Although the function of the glycan moiety of these proteins has yet to be determined, there are a number of reports describing a role in virulence, colonization and macromolecular assembly [33-35].

Two different mechanisms for protein glycosylation have been observed and are based on the mechanism through which the glycans are transferred to proteins. In the first mechanism, the transfer of carbohydrates occurs directly from nucleotide-activated sugars to acceptor proteins. This mechanism is typical of $O$-glycosylation in eukaryotes, and flagellar glycosylation in several bacterial species [9]. Alternatively, an oligosaccharide can be preassembled on a polyisoprene carrier before being transferred en bloc to protein acceptors by an oligosaccharyltransferase. This type of mechanism is found in the $N$-glycosylation pathways in eukaryotes, bacteria [36] and archaea, and $O$-linked glycosylation of pili in $P$. aeruginosa [27,37,38] and Neisseria species [21]. Of particular note, the $N$-linked glycosylaton pathway of $C$. jejuni produces a conserved heptasaccharide with the structure GalNAc$\alpha 1,4-G a l N A c-\alpha 1,4-(G l c-\beta 1,3)-G a l N A c-\alpha 1,4-$ GalNAc- $\alpha 1,4-G a l N A c-\alpha 1,3-B a c 2,4$ diNAc- $\beta 1$, where Bac2,4diNAc is 2,4-diacetamido-2,4,6trideoxyglucopyranose [39] and is assembled by proteins encoded in the protein glycosylation ( $p g l$ ) locus [16]. This glycan is synthesized on a polyisoprenyl carrier at the cytoplasmic face of the inner membrane through the action of a phosphoglycosyltransferase (PglC) and several glycosyltransferases (PglA, PglH, PglI and PglJ). The assembled oligosaccharide is then flipped to the periplasm by the action of the membraneassociated flippase $(\mathrm{PglK})$. Once in the periplasm, the oligosaccharide is transferred to proteins that possess the extended sequon Asp/Glu-XAsn-Y-Ser/Thr (where $\mathrm{X}$ and $\mathrm{Y}$ are any amino acid except Pro) by the oligosaccharyltransferase (PglB) $[40,41]$. The similarities between the Pgl pathway in Campylobacter and the eukaryotic $N$-glycosylation machinery have resulted in an explosion of research on this pathway, as the pathway serves as a simplified model for studying the eukaryotic system. Additionally, it can be functionally transferred to E. coli [42], where it shows potential for applications in glycoengineering [43] .

Detection of bacterial glycoproteins is often a challenging task owing to the diverse array of monosaccharide building blocks available to bacteria. Nevertheless, detecting novel bacterial glycoproteins and predicting the structure of the attached glycans is virtually impossible, and must be deduced using a wide range of bioanalytical techniques. These include high-performance liquid chromatography (HPLC) [44], mass spectrometry (MS) [45-48], nuclear magnetic resonance (NMR) [49], polyacrylamide gel electrophoresis (PAGE) [50] and capillary electrophoresis (CE) [51-53]. Typically studies commence with glycoprotein detection by $1 \mathrm{D}$ or $2 \mathrm{D}$ gel electrophoresis, where aberrant protein migration is often the first clue. Commercially available lectin-binding kits or carbohydrate-reactive dyes are frequently used. Putative glycoproteins are then typically isolated and their initial characterization generally relies heavily upon MS, exploiting so-called 'top-down' or 'bottom-up' techniques. An outline of these approaches, as applied to bacterial glycoprotein discovery and analysis, is presented in Figure 2 . The higher throughput study of the entire complement of glycoproteins within a bacterial cell is an emerging field known as 'glycoproteomics', a description of which is beyond the scope of this article. Progress in bacterial glycoproteomics has recently been reviewed [54].

'Bottom-up' MS analyses of putative bacterial glycoproteins can be carried out on purified glycoprotein(s) or protein(s) separated by gel electrophoresis. The protein is digested with 


\section{Review Reid, Fulton \& Twine}

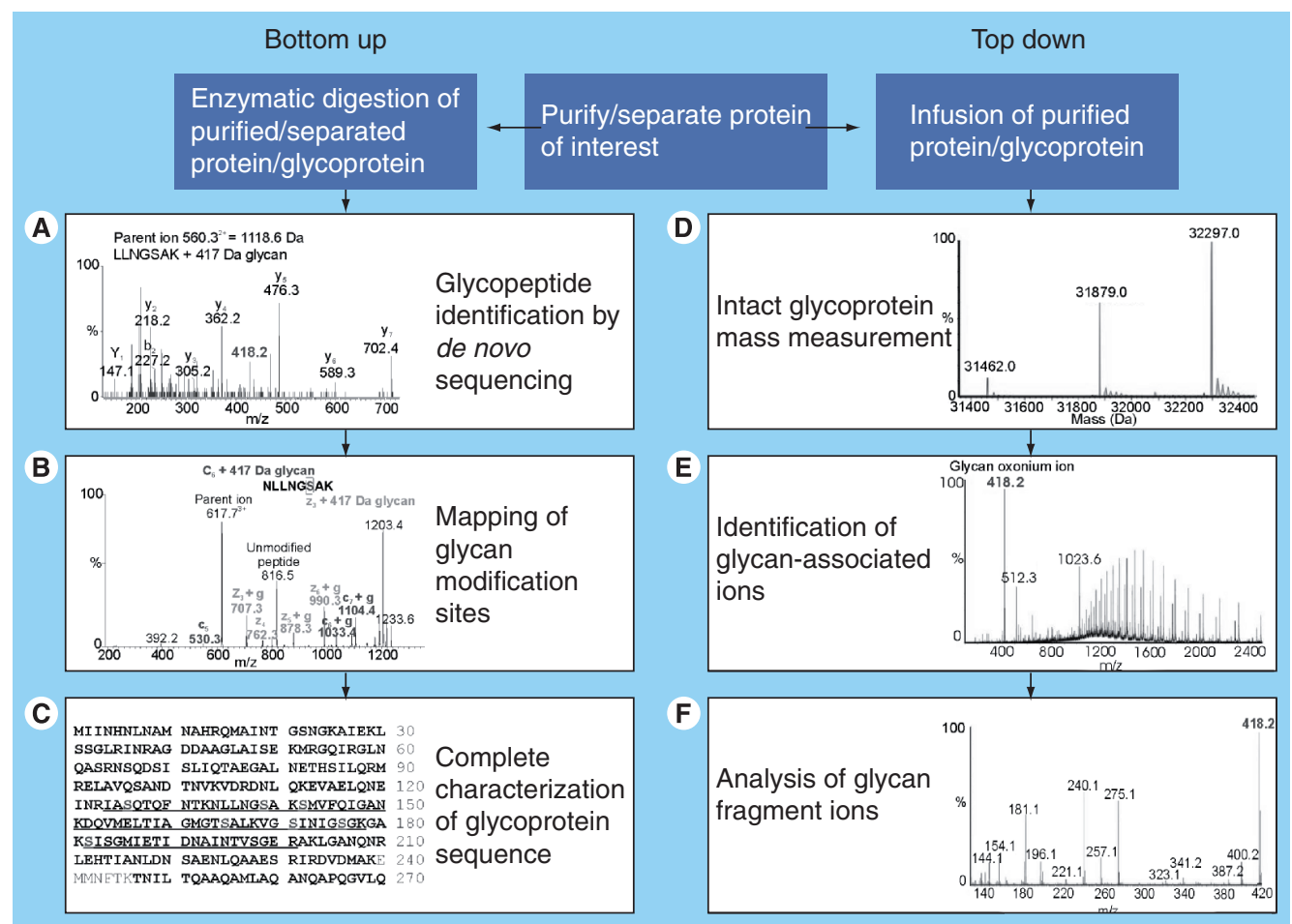

Figure 2. Mass spectrometry workflow for the identification and characterization of individual bacterial glycoproteins. 'Bottom-up' analyses of glycoproteins at the peptide level: the bottom-up method involves the tandem mass spectrometry (MS/MS) analysis of enzymatically digested protein/glycoprotein. (A) The diverse and unusual bacterial glycans make the interpretation of bacterial glycopeptide MS/MS data challenging. De novo peptide sequencing is often required for identification of bacterial glycopeptides. (B) Mass spectrometers equipped with electron transfer dissociation (ETD) or electron capture dissociation (ECD) can be used to determine individual sites of glycan linkages in glyopeptides. Both ETD and ECD afford an alternative method of peptide fragmentation to more widely used collision-induced dissociation, preserving delicate glycan modifications; (B) shows a representative ETD spectrum of Clostridium botulinum glycopeptide. A combination of these complementary techniques allows a comprehensive characterization of a putative glycoprotein sequence. (C) Typical peptide sequence coverage obtained for FlaA protein from MS/MS and ETD data. These data showed that glycan was attached through $O$-linkage, with seven sites of modification (Ser126, Ser139, Ser142, Ser165, Ser171, Ser176 and Ser182). 'Top-down' MS analyses of purified intact glycoproteins: the top-down approach uses mass spectrometry techniques to analyze the intact protein. (D) An observed mass in excess of the mass expected based on the translated genome sequence is often indicative of post-translational modification, such as glycosylation. The MS-profile of an intact protein usually results in a broad envelope of multiply charged protein ions in the MS spectrum, from which the reconstructed molecular mass profile can be calculated. The data shown in (D) shows the observed masses of FlaA from C. botulinum Alberta, with three distinct masses of 32297, 31879 and $31462 \mathrm{Da}$. Of note, each of the major intact mass peaks was separated by a mass of $417 \mathrm{Da}$, which corresponds to the mass of the flagellin glycan. MS/MS analysis of a single, multiply charged protein ion, can often yield information regarding the masses of labile, protein-associated, glycan ions. Analysis of $C$. botulinum Alberta flagellins using this method revealed at least two distinct marker ions of mass-to-charge ratio 512.3 and 418.2. By adjusting the collision energy at the front end of the mass spectrometer, these glycan associated ions can also be observed (known as front-end collision-induced dissociation) (E). Subsequently, MS/MS of the observed glycan fragment ions can yield information about the glycan composition. For the FlaA protein of $C$. botulinum, the spectrum of the glycan oxonium ion at mass-to-charge ratio 418 gave an MS/MS spectrum with predominant peaks at 274, 240 and $181 \mathrm{Da}$. The fragmentation pattern of this glycan moiety closely resembled that previously observed for pseudaminic acid or legionaminic acid, with an additional unknown mass of $143 \mathrm{Da}$. Overall, these data showed the FlaA protein to be modified with a pseudaminic acid-like sugar of $417 \mathrm{Da}$, at seven sites via $O$-linkage via serine residues. The structure of this sugar was determined by nuclear magnetic resonance of a purified glycopeptide, and shown to be a novel legionaminic acid derivative, 7-acetamido-5-( $N$-methyl-glutam-4-yl)-amino3,5,7,9-tetradeoxy-d-glycero- $\alpha$-D-galacto-nonulosonic acid [13] .

Data represented uses Clostridium botulinum strain Alberta flagellin, FlaA, as a representative glycoprotein [13]. 
a proteolytic enzyme, most commonly trypsin, and analyzed by tandem MS (MS/MS). Consequently, strategies to rapidly identify and evaluate glycopeptides are required. The use of software tools for assignment of peptide fragmentation data are widely used as a matter of routine (e.g., MASCOT ${ }^{\text {тм} ; ~ M a t r i x ~ S c i e n c e, ~ L o n d o n, ~}$ UK). By contrast, the development and use of bioinformatics tools and databases for glycobiology and glycomics research has been slow. Softwareassisted data analysis of bacterial glycopeptide tandem mass spectra is often hampered by a lack of knowledge of the glycan produced by a particular bacterium. Therefore, de novo glycopeptide analyses are necessary, despite being time consuming and often a bottleneck in such studies. Additional information obtained from 'bottom-up' studies includes linkage site information and glycan sequence information [55]. Observation of glycan MS/MS fragmentation patterns can potentially provide clues as to the glycan structure.

'Top-down' MS permits the study of the intact protein, rather than the peptides generated from proteolytic digestion of the protein. Recently, a top-down MS approach was applied to expedite the identification of unusual bacterial glycoprotein-associated ions (so-called 'oxonium' ions) [56]. The selectivity and specificity of this approach was exploited for the identification of the unusual glycan modification of flagellin from protein $H$. pylori [56]. Studies of Clostridium botulinum strains also used this approach as a higherthroughput method of characterizing flagellin from a wide range of $C$. botulinum isolates, and showed potential for flagellin glycan-associated marker ions in strain differentiation [12,57].

Newer strategies are currently being developed to specifically enrich glycoproteins or peptides from bacteria. Some of these approaches are being adapted from methods developed for the study of eukaryotic glycans [58]. For example, hydrazide capture of $N$-linked glycoproteins, lectin affinity purification and ion-pairing normal phase liquid chromatography (IP-NPLC) are being applied to prokaryotic glycoprotein discovery, and are increasing the study of glycoproteomics $[59,60]$.

Of particular interest, a recent study used IP-NPLC to resolve glycosylated peptides from a tryptic digest of $C$. jejuni 11168 periplasmic proteins. The method used acids as ion-pairing reagents in normal-phase chromatography to increase the hydrophobicity differences between nonglycopeptides and glycopeptides. This permitted the reproducible isolation of bacterial $N$-linked glycopeptides, modified with nonsialylated heptasaccharides, and expression comparisons of glycopeptides between C. jejuni 11168 and its $p g l D$ mutant [59]. This study reported the first observation of heptasaccharides containing monoacetylated bacillosamine-modifying C. jejuni glycoproteins [59].

Identification of glycoproteins is the first challenge; the second is gathering structural information regarding the attached sugar. In many cases, this requires release of the glycan from the protein, to allow detailed structural analyses of the glycan using MS or NMR. Release of bacterial glycans from proteins can be challenging - many existing methods were developed for release and study of eukaryotic glycans. These techniques may not be applicable to bacterial proteins because the carbohydrate-peptide linkages are different and the enzymes capable of releasing the carbohydrate moieties are rarely available. However, a universal glycomics method was used to address the challenges of glycan structural investigations. This method, based on the combination of nonspecific proteolytic digestion and permethylation, was applied as a complementary approach to study the diversity of C. jejuni $11168 N$-linked sugars [61]. $N$-glycome analysis of $C$. jejuni 11168 allowed, for the first time, detection of free heptasaccharide that were unexpectedly produced by the $N$-glycan pathway within the periplasm. The authors were able to clearly distinguish between free glycan and asparagine-linked glycan [61].

Unambiguous structural assignment is most readily obtained from NMR studies of purified sugars or short glycopeptides. In addition to challenges in releasing glycans from bacterial proteins, obtaining sufficient glycan material from purified proteins is difficult. In attempts to overcome this obstacle, focused metabolomics studies have targeted intracellular nucleotideactivated glycan precursors in order to characterize glycan biosynthetic pathways, using both MS and NMR. Such studies have been pivotal in the structural analysis of $C$. jejuni proteinassociated glycans, and in functional studies of the associated biosynthetic pathways [17,62-67]. Such work is often a prerequisite for further functional studies of the role of the glycoprotein in virulence.

\section{MS \& related technologies in bacterial nonprotein glycan discovery Detection of bacterial glycolipids by MS}

Technological advancements in genomics, transcriptomics and proteomics have allowed scientists to probe biological systems with increased 
precision and sensitivity. While the analysis of lipids from microorganisms has been around since the 1950s, the tools for the examination of the lipidome and, in particular, the glycolipidome are still emerging [68-70]. The role of glycolipids in host-pathogen interactions is an emerging field, with the bulk of the scientific literature revolving around the analysis of lipid A [71,72]. Traditional methods for the analysis of lipid-linked oligosaccharides (LLOs), a subclass of biologically relevant glycolipids, usually involves chemical or radioactive labeling of the oligosaccharide component followed by chromatographic or electrophoretic separation [73]. In the realm of bacterial glycolipidomics, there is an increasing body of work on the assembly of bacterial polysaccharides and the polyisoprenyl carriers upon which they are assembled. MS has been the tool of choice to investigate the levels of undecaprenyl phosphate in bacterial membranes [74], levels of lipid II, an intermediate in peptidoglycan biosynthesis [75], and levels of polyisoprene intermediates in LPS biosynthesis in Salmonella spp., E. coli and Francisella novicida [76-78]. While glycolipidomics is still an emerging field, the C. jejuni $\mathrm{Pgl}$ pathway (described in detail previously) will be used as an example of the information that can be obtained by analyzing lipid-mediated steps in oligosaccharide biosynthesis in vivo.

Lipooligosaccharide profiling of the Pgl pathway using the newly developed affinity-capture CE-MS has provided unique insight into the synthesis of the $N$-glycan oligosaccharide [79]. This method has allowed for the rapid enrichment of LLOs, utilizing an immobilized lectin (soybean agglutinin) with specificity towards the GalNAc residues in the C. jejuni LLO of interest. In order to characterize the membrane-associated steps in the Pgl pathway, both wild-type C. jejuni and isogenic mutants of the pathway were used to investigate the assembly of LLO intermediates. Analyses of isogenic mutants in the oligosaccharyltransferase $(p g l B)$ and membrane flippase $(p g l K)$ revealed an accumulation of undecaprenyl-pyrophosphate (Und-PP) heptasaccharide, with a significantly greater amount found in the flippase mutant. The lower levels of Und-PP-heptasaccharide in the $p g l B$ mutant is most likely due to the presence of enzymes in the periplasm responsible for the recycling of Und-PP. The accumulation of LLO intermediates in these mutants confirmed that the C. jejuni $\mathrm{N}$-glycan is assembled on undecaprenyl phosphate. Analysis of isogenic mutants in steps upstream of the flippase PglK revealed an absence of accumulating truncated LLO intermediates, suggesting feedback regulation of the pathway. This conclusion was supported by the observation of the accumulation of UDP-diacetamido-trideoxy-Hex in the galactosyltransferase mutants, $p g l J$ and $p g l H$, and the observed allosteric regulation in the analogous yeast oligosaccharyltransferase complex [80]. Since undecaprenol is in limited abundance in the bacterial cell and is involved in a plethora of cellular activities, some of which are vital for cell survival, it is not surprising that a feedback regulation of the Pgl pathway would exist to prevent the accumulation of LLOs and sequestering of undecaprenol from other biological processes.

The application of affinity capture-MS to the analysis of membrane-associated processes in carbohydrate biosynthesis pathways provides a simple and rapid method to gather structural information for the oligosaccharides under study. Unlike conventional techniques, radioisotope labeling and chromatographic separation are not required for analysis [81]. In addition, by varying the lectin used for affinity capture, this method can be applied to a wide range of systems. Analysis of complex bacterial carbohydrate biosynthetic pathways, such as those for pilin $O$-glycosylation and peptidoglycan assembly, may provide new insights into the synthesis and control of these glycans. Targeted glycolipidomic profiling is a powerful tool for bacterial glycobiology. It provides a high-throughput method for assessing the role of genes in a biosynthetic pathway and allows for the identification of potential regulatory mechanisms, by detecting the accumulation or absence of intermediates in the pathway. With the recent advances in glycoengineering in bacteria, affinity-capture CE-MS also allows for a more comprehensive assessment of the efficiency and potential bottlenecks associated with producing potential protein glycoconjugates in bacteria. Current work on the analysis of bacterial LLOs is focused on the development of more general and nonselective enrichment procedures.

\section{LPS profiling by CE-MS}

Capillary electrophoresis is a high-resolution technique for the separation of complex biological mixtures [82]. When coupled with MS, CE provides a powerful approach for the identification and structural characterization of analytes. This technique has been used for the characterization of bacterial glycolipids and glycoconjugates from bacteria, including $H$. influenzae [83-87], Neisseria meningitidis [85,88], Pasteurella 
multocida [89-91], H. pylori [92,93], C. jejuni [94-96], Vibrio anguillarum [97] and Aeromonas salmonicida [98]. CE-MS has been widely used for characterizing differences in LPS glycoform and phosphorylated populations $[84,88,99]$. This is achieved by exploiting differences in the molecular conformations and charge distributions of closely related LPS molecules during electrophoretic separations.

A recent study employed immunoaffinity capture CE-MS to investigate LPS changes in the fish pathogen $A$. salmonicida under co-culture conditions [100]. When co-cultured with trout macrophages in intraperitoneal chambers, significant differences were observed in the mass spectral fragmentation patterns of the LPS $O$-chain polysaccharide isolated in vivo. The $O$-antigen repeating unit was previously reported to contain a rhamnose residue substituted with side chain glucose and $O$-acetyl group [101]. Compared with LPS isolated from cells grown in vitro, glycoforms containing multiple glucose residues and $O$-acetyl groups were observed in the in vivo samples.

Lipopolysaccharide is implicated as a major virulence factor of $H$. influenzae. Nontypeable H. influenzae (NTHi) is a significant cause of otitis media in children, and is the most common pathogen recovered from the middle ear of children with recurrent episodes [102]. Although immunity against NTHi appears to develop after acute otitis media, protection is strainspecific, permitting recurrent episodes owing to antigenically distinct isolates. Extensive structural studies of LPS from $H$. influenzae have led to the identification of a conserved glucose-substituted triheptosyl inner-core linked to lipid A [103-109]. The LPS of $H$. influenzae mimics host glycolipids and possesses several phase-variable modifications, which allow the bacterium to evade the host immune system. Recent work has shown that NTHi LPS oligosaccharides replaced by terminal sialic acid ( $N$-acetyl neuraminic acid) residues are critical virulence factors in the pathogenesis of otitis media in chinchillas [110] and gerbils. In the chinchilla, sialyation of LPS modifies the interaction with complement [111]. This altered recognition could be attributed to sialylation masking the LPS epitopes recognized by complement [111] or molecular mimicry of host-like structures [110]. It has also been proposed that the expression of sialylated LPS glycoforms may play a role in pathogenesis by promoting biofilm formation [112-114]. One study, using a CE electrospray ionization MS (CE-ESIMS) technique on NTHi isolated from chinchilla, suggested that the virulence afforded by LPS sialylation depends on the ability of NTHi to scavenge sialic acid from the host [110]. Further work on NTHi LPS glycoforms followed changes in LPS up to 7 days postchallenge in the chinchilla and constituted the first detailed description of LPS glycan patterns during the course of a bacterial infection [115]. Over the course of the study, a trend toward less-complex glycoforms was observed as the infection progressed. It was suggested that the organisms responsible for maintaining active infection may undergo major changes in their metabolic profile as a consequence of available nutrients and their response to the stresses mediated by the host's plethora of innate and acquired immune responses [112]. The biosynthesis of shortened LPS glycoforms may represent a metabolic consequence or an adaptive response to limited energy resources available within the host environment [115]. The role of the in vivo LPS $O$-antigen in pathogenesis is not clear, but it is interesting that the LPS carbohydrate structure is influenced by the host environment. This underlines the importance of further studies of LPS and other glycans from bacteria grown within the host environment.

One drawback to LPS analysis by CE-MS is the limitations posed by the buffer used in CE separation, and the deleterious effects it can have on detection limits. Gas-phase ion mobility separations have the potential to separate glycan structural isomers, based upon their 3D shape $[116,117]$. High-field asymmetric waveform ion mobility spectrometry (FAIMS) is a gas-phase ion separation technique based on compound-dependent differences in ion mobility at high field $[118,119]$. The FAIMS system acts as an ion filter which continuously transmits a selected type of ion, independent of mass-tocharge ratio. This allows users to selectively isolate target ions based upon physical properties. The approach also reduces chemical background and other interferences in MS spectra. FAIMS is increasingly being combined with front end LC or CE separations. The coupling of a FAIMS to a CE-MS system was successful in separation of trace levels of LPS oligosaccharide glycoforms from NTHi [83]. It was found that the signal of interest was increased as many as 7.5-fold when the FAIMS device was used [83].

\section{Bioinformatics for glycomics}

The development and use of informatics tools and databases for glycobiology and glycomics research has increased considerably in recent years. However, the general development in 
this field can still be considered as being in its infancy, when compared with the bioinformatics tools available to the genomics and proteomics communities. For example, the automated identification of peptides in proteomics studies is becoming almost routine, with the use of tools such as Mascot [301]. The major factor hindering the development of equivalent tools for glycoproteomics and glycomics is the lack of automated tools that can retrieve structural information from MS data. This challenge is compounded by the structural diversity of glycans and the variety of methods required to characterize both glycopeptides and the modifying glycan. The development of library-based glycan sequencing tools are slowed by the lack of well-curated collections of glycan sequences.

Presently, MS-based glycan and glycopeptide analysis is generally restricted to specialist laboratories that posses the expertise required for manual interpretation of glycan-containing spectra. Even for trained mass spectrometrists, analysis of multiple glycan MS or MS/MS spectra frequently becomes the bottleneck in data analysis. In many cases, manual annotation can be resource limiting or even prohibitive for a high-throughput glycomics screen. Tools are being developed to address issues in glycan profiling, particularly the recent development of an algorithm called Cartoonist, which performs automated annotation of MALDI-TOF spectra [120]. This works with single MS spectra to determine the composition of a particular glycan structure. Cartoonist assigns plausible glycan structures and provides a confidence level assignment to aid further analysis. The graphical forms consist of the spectra annotated with either cartoons of the structures in symbol form, or with formulas of each monosaccharide.

Other algorithms and tools for either glycan structure prediction or glycan annotation also exist. For example, the GLYCH method was developed for the interpretation of MS data, to list all possible fragment types of glycans [121]. Other online tools for annotating glycan structures from mass peaks include GlycoPep ID [122], Glyco-Mod [123] and GlycoPeakFinder [124].

In addition to interpretation tools, there are currently three major databases that house glycan structure data: KEGG Glycan [125], Glycosciences.de [126] and a relational database hosted by the Consortium for Functional Glycomics (CFG) [127], an international initiative funded by the National Institute of General Medical Sciences (MD, USA). The CFG's database is a web portal connected to the integrated interfaces of diverse datasets in the CFG's relational databases, which contain information regarding glycan-binding proteins, glycan structures and associated glycosyltransferases. The Kyoto Encyclopedia of Genes and Genomes database (KEGG) have produced an extension, KEGG Glycan, which is managed and developed by the Kyoto Unversity Bioinformatics Center (Kyoto, Japan). Glycosciences.de is maintained by the German Cancer Research Center (Heidelberg, Germany) and provides researchers with MS and glycan structure data, as well as applications for glycan analysis. Although the three current databases share the same initial collection of glycan structure, they use different file formats, which, in proteomics and glycoproteomics alike, is a huge informatics stumbling block. GlycoVault is a web-based informatics gateway that contains databases, ontologies and other glycan structure-related data. This application also contains the Glycomics Browser, a webbased visualization and analysis tool for glycan data. The use of these databases in the analysis of bacterial glycomes will rely upon the continued accumulation of bacterial glycan structural information. For further information, readers are directed to an excellent recent tutorial article [128].

\section{Application of NMR to bacterial glycomics}

Nuclear magnetic resonance, in particular liquid state NMR, has been employed to study microbial metabolism for more than 30 years [129], and has played a critical role in the determination of the structure of a myriad of microbial glycoconjugates. NMR has been fundamental for the structural analysis of CPS and LPS from numerous microbial pathogens [130-138]. While NMR has provided detailed information regarding the biomimicry of these molecules, this review will focus on the application of whole-cell and solid-state NMR to the elucidation of the pathogen glycome.

\section{HR-MAS whole-cell NMR}

High-resolution magic angle spinning NMR (HR-MAS NMR) was initially developed in the late 1990s and has been used to characterize a variety of biological samples $[139,140]$. In HR-MAS NMR, the sample is spun at the magic angle of $54.7^{\circ}$ in order to reduce spectral line broadening caused by chemical shift anisotropy and contributions from magnetic susceptibility [141,142], making this technique amenable to the acquisition of high-resolution spectra from various biological matrices, including intact bacterial cells. The majority of the 
literature on HR-MAS whole-cell NMR has focused on the CPS of Gram-negative pathogens, such as $C$. jejuni [96] and N. meningitidis [143]. In recent years, HR-MAS whole-cell NMR has been expanded to cell wall components of mycobacteria, such as arabinogalactan [144].

High-resolution magic angle spinning NMR has been used extensively to study the CPS and the $N$-linked Pgl pathway (described previously) in the intestinal pathogen $C$. jejuni $[40,145,146]$. The CPSs produced by $C$. jejuni are important virulence factors involved in colonization and invasion and are the major antigenic component of Penner's serotyping system [147]. To date, there have been several $C$. jejuni $\mathrm{CPS}$ structures reported that identify a number of phase-variable structural variations, such as the incorporation of methyl, ethanolamine and aminoglycerol groups on the CPS sugars [148,149]. One of the more unusual modifications reported is the $O$-methyl phosphoramidate $\mathrm{CH}_{3} \mathrm{OP}(\mathrm{O})\left(\mathrm{NH}_{2}\right)$ (OR) (MeOPN) group - a labile, phase-variable phosphorylated structure. HR-MAS NMR has been employed as a rapid, high-throughput means to directly examine animal and human isolates from diverse clinical presentations and geographical locations for the presentation of the MeOPN CPS modification [150]. An interesting aspect of this study by McNally et al. was the application of HR-MAS whole-cell NMR to examine $\mathrm{MeOPN}$ expression within an avian host [150], providing evidence that this phasevariable modification is present during colonization of the GI tract (FIgUre 3). In addition, this technique was used to demonstrate the commonality of this phase-variable modification in 61 of 111 strains tested. Through the combination of bioinformatic analysis of the $C$. jejuni genome, mutagenesis and whole-cell NMR, the authors were able to identify the genes responsible for the biosynthesis of this unique modification. The applicability of HR-MAS whole-cell NMR as a high-throughput screen for identifying genes involved in the biosynthesis of surface polysaccharides is further illustrated by the identification of the genes responsible for D-glycero-Lglucoheptose biosynthesis in C. jejuni 11168 CPS [145].

The applicability of HR-MAS NMR is not restricted solely to the analysis of surface polysaccharides. It has also served to elucidate the $N$-linked Pgl pathway in C. jejuni 11168 [96]. Through the use of a CPS-negative strain, whose signals interfere with the detection of $N$-glycan signals, the anomeric signals of the $N$-glycan heptasaccharide were observed in vivo. This

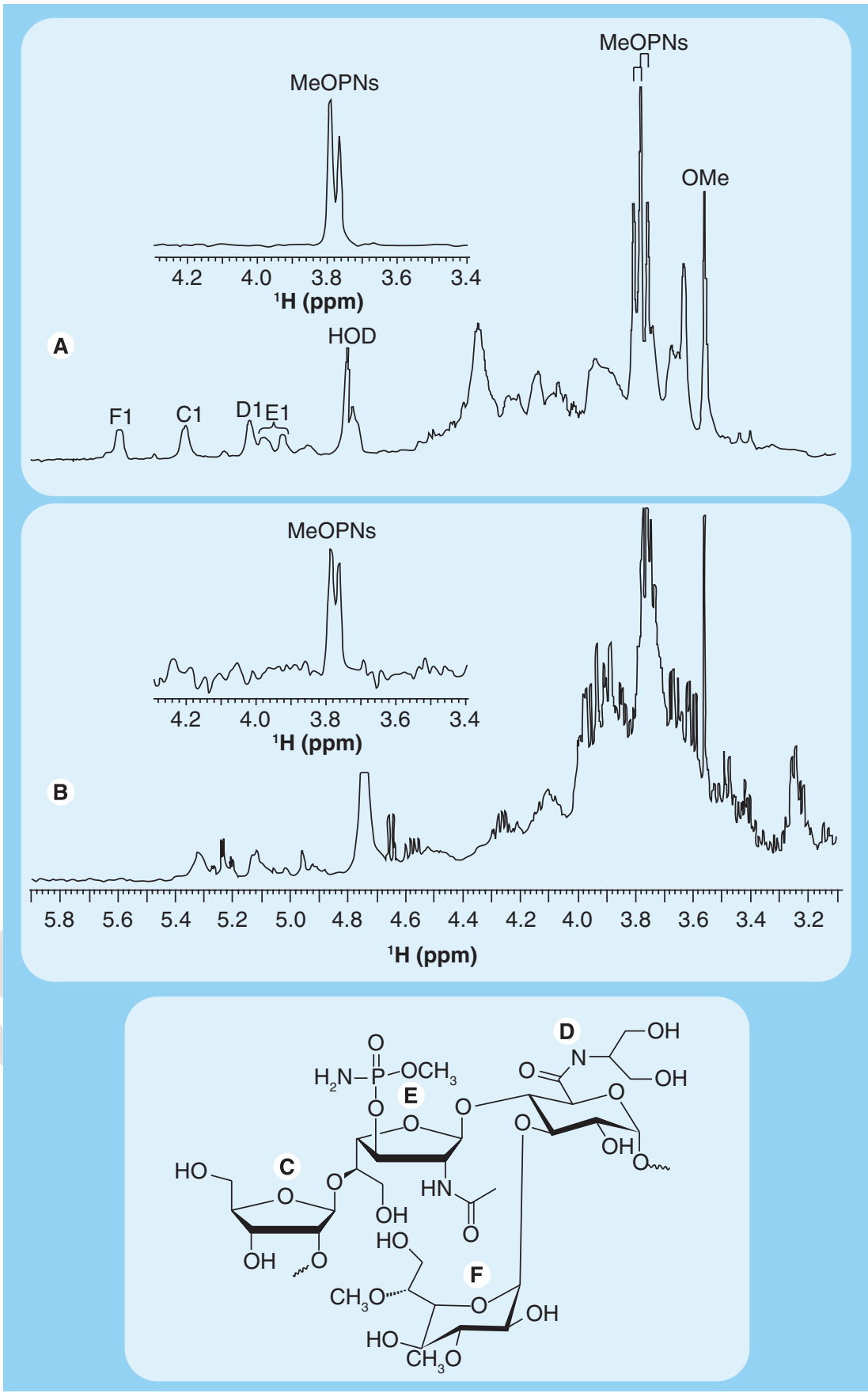

Figure 3. High-resolution magic angle spinning nuclear magnetic resonance analysis of the cecal contents of a representative Campylobacter jejuni colonized chick. (A) High-resolution magic angle spinning (HR-MAS) ${ }^{1} \mathrm{H}$ nuclear magnetic resonance (NMR) spectrum of plate-grown $11168 \mathrm{H}$ cells (256 scans). The inset shows the spectrum for the same sample analyzed with a ${ }^{1} \mathrm{H}_{-}{ }^{31} \mathrm{P}$ heteronuclear single quantum coherence HR-MAS NMR experiment (1024 scans, ${ }^{31} \mathrm{P}$ decoupled, ${ }^{1} \mathrm{~J}_{\mathrm{H}, \mathrm{P}}=12 \mathrm{~Hz}$ ). (B) The HR-MAS ${ }^{1} \mathrm{H}$ NMR spectrum of the cecal contents of a chick that has been colonized with $\mathrm{C}$. jejuni $11168 \mathrm{H}$ at $48 \mathrm{~h}$ post-inoculation (256 scans). The inset shows the spectrum for the same cecal sample examined using the ${ }^{1} \mathrm{H}-{ }^{31} \mathrm{P}$ heteronuclear single quantum coherence HR-MAS NMR experiment (1024 scans). OMe is the $-\mathrm{OCH}_{3}$ group located at $\mathrm{C}-6$ of residue (F) and HOD is the deuterated water resonance. All spectra were acquired at $500 \mathrm{MHz}\left({ }^{1} \mathrm{H}\right)$. The labeling of resonances $(\mathbf{C}-\mathbf{E})$ correspond to the anomeric signals of the monosaccharides in the $11168 \mathrm{H}$ capsular polysaccharide structure presented at the bottom. Reproduced with modifications from [150]. MeOPN: O-methyl phosphoramidate. 
provided the capacity to quantify the levels of $N$-glycan heptasaccharide in isogenic mutants of the Pgl pathway, and assess the ability of the oligosaccharyl transferase PglB to transfer truncated glycans. It should be noted that the ability to observe the $N$-linked glycan by HR-MAS NMR is most likely due to the production of protein-free heptasaccharide in the periplasm, resulting from competition between the transfer of the lipid-linked heptasaccharide to protein, and hydrolysis by water [61].

While the vast majority of applications of HR-MAS NMR to glycomics have utilized C. jejuni, there have been several applications of this technique to the study of other microbial glycomes, particularly in the structural characterization of the cell wall of living mycobacterial cells [144,151]. In these studies, Mycobacterium smegmatis was used as a model organism for the pathogen $M$. tuberculosis in an attempt to elucidate the major carbohydrate components of the mycobacterial cell wall. The major challenges in studying the mycobacterial cell wall revolve around deciphering the cell wall biosynthetic pathways and its interaction with antimycobacterial drugs and host tissues. From a 'systems biology' viewpoint, identifying how the various cell wall components interact with each other could facilitate the development of new anti-TB drugs and treatment strategies. Lee and coworkers have successfully applied multidimensional HR-MAS NMR to the analysis of whole-cell mycobacteria $[144,151]$. The use of selective labeling techniques, such as heavy nitrogen $\left({ }^{15} \mathrm{~N}\right)$ and carbon $\left({ }^{13} \mathrm{C}\right)$ sources has allowed for the resolution of signals from different mycobacterial macromolecular structures, including mycolyl arabinogalactan, lipoarabinomannan and glycolipids. Through the use of selective labeling, the authors were able to measure the levels of arabinogalactan and lipoarabinomannan in a semi-quantitative manner, which is a useful feature when investigating the biosynthetic pathways of these molecules. An interesting feature of using multidimensional HR-MAS NMR is the ability to utilize a whole-systems approach to study the effects of antibiotics on the mycobacterial cell wall. In the case of $M$. smegmatis, the effects of ethambutol (EMB), a first-line treatment for TB known to reduce the arabinan content of the cell wall through inhibition of the arabinosyltransferases EmBA-C, were investigated [152]. HR-MAS NMR indicated a significant decrease in most arabinose signals in the mycobacterial cell wall upon treatment with EMB, with the exception of the 5'-linked Araf in the arabinogalactan core, suggesting that EMB inhibits multiple enzymes in the arabinan biosynthetic pathway, but not the arabinosyltransferase, which builds the $5^{\prime}$-linked core [144]. This approach allows for the observation of the effects of drug treatment and gene mutation on the cell wall, which could help in defining mechanisms of action for other cell wall inhibitors, and the screening of new cell wall-acting drugs. It will be interesting to see if this methodology can be applied to the elucidation of the role of the mycobacterial cell wall in host cell interactions.

\section{Solid-state NMR of peptidoglycan}

Another component of the bacterial glycome that can sometimes be overlooked owing to its inherent heterogeneity and insolubility is peptidoglycan. With peptidoglycan serving as a major structural component of the bacterial cell, studying the biosynthesis, metabolism and dynamics of this critical structure by traditional techniques can be difficult. While advances have been made in the characterization of peptidoglycan-acting enzymes with their peptidoglycan substrates $[153,154]$, these are in vitro techniques that cannot begin to dissect the complex interactions that occur with such a heterogeneous substrate. In addition, peptidoglycan biosynthesis and metabolism has traditionally been a well-spring of antimicrobial targets, and still holds vast potential for the development of new antimicrobials. Recent applications of solid-state NMR to the study of the dynamics and structure of the Gram-positive cell wall demonstrate the potential of this technique for studying peptidoglycan dynamics in vivo [155], as well as for dissecting the mode of action and binding specificity of cell wall-acting antibiotics [156-161].

To date, solid-state NMR of Gram-positive cell walls has predominantly focused on the analysis of the stem peptide and pentapeptide bridges linking adjacent glycan strands in Staphylococcus aureus [155]. Through the use of ${ }^{13} \mathrm{C}$ - and ${ }^{15} \mathrm{~N}$-labeled amino acids typically found in the peptidoglycan stem peptide, a quantitative measurement of the degree of cross-linking could be obtained in intact $S$. aureus cells. Traditional methods for analysis of peptidoglycan crosslinking usually involve enzymatic digestion and analysis of muropeptides by HPLC or MS [162]. The use of solid-state NMR and labeling experiments provided information regarding the tertiary structure of the peptidoglycan sacculus [163]. Spin diffusion experiments can be used as a molecular ruler to measure the distance between the glycan backbone and stem peptide, 
which provided information on the orientation of peptidoglycan strands in the Gram-positive cell wall. The results obtained from these experiments bring into question the traditional view of Gram-positive cell wall architecture. The commonly held view is that the glycan backbone adopts a helical twist so that the stem peptides within a plane perpendicular to the glycan main chain are antiparallel $[162,164,165]$. The use of solid-state NMR and spin diffusion studies seems to suggest that the stem peptides can be antiparallel as well [166]. This in vivo analysis of peptidoglycan provides relevant data on the organization of this essential bacterial polysaccharide under physiologically relevant conditions. Solid-state NMR provides a powerful tool to assess the interaction of potential antimicrobial candidates developed to target the bacterial cell wall $[158,159,166-168]$ and provides direction in the refinement of activity or target specificity for medicinal chemists.

\section{Probing the bacterial glycome using chemical \& physical methods Chemical labeling: bioengineering of bacterial glycans}

Sialic acids have been shown to be involved in many host-pathogen recognition events. As a result, there has been much activity in the search for methods to enrich for and identify sialation in complex tissues [169-172]. Studies have demonstrated that analogues of glycan precursors, for example azido analogues of sugar precursors, can succesfully be incorporated in living cells [171-184]. The resulting azido sugars, expressed on the cell surface, can then be labeled via reactions such as the Staudinger ligation (commonly referred to as 'click chemistry'), functioning as a bio-orthogonal reporter group [175]. This type of approach is being exploited for the study of both eukaryotic and bacterial cells [172]. For example, the pseudaminic acid (Pse) pathway of $C$. jejuni has been targeted using this type of approach. Pse is a nine-carbon $\alpha$-keto acid that is structurally related to the sialic acids. The sugar is $O$-linked to flagellin proteins in pathogenic bacteria, such as $H$. pylori and C. jejuni. Glycosylation is required for assembly of the flagellar filament, and has been implicated in the virulence of these bacteria [176].

In a recent report, Liu and coworkers synthesized a neutral azido sugar precursor that was taken up by $C$. jejuni cells and converted into an azido-labeled Pse derivative in vivo [177]. The third intermediate in the Pse pathway, 6-deoxyAltdiNAc, was targeted for the introduction of the labeled analogue, 6-deoxy-Alt-NAc4NAz, based on synthetic considerations. The analogue was incorporated into the growth medium, where it competed readily with the natural substrate and was tolerated by downstream steps in the Pse pathway. The bacteria harboring the azido-labeled Pse were motile and the azido functionality was maintained after in vivo incorporation into flagella. While the use of these tags in glycomic imaging experiments has been reported for eukaryotic systems [178,179], applications for these modified glycans in bacteria have yet to be demonstrated.

Lectin microarrays: the potential for dynamic studies of the bacterial glycome

A lack of high-throughput analytical tools for the characterization of bacterial glycans has been an obstacle in the examination of the dynamics of bacterial carbohydrate variation over the course of infection. Recently, lectin-based microarrays have been developed as a glycoprofiling tool. This technology exploits many features of more traditional microarray tools, but with a series of lectins immobilized on a single chip, allowing high-throughput screening of the entire cellular glycome [180-182]. Lectins are naturally occurring glycan-binding proteins [183]. A wide variety of lectins has been described, each with a unique glycan-binding profile. Some lectins bind to a very specific glycan structural feature, while others show a wider or more complex binding profile [184]. The commercial availability of purified lectins has led to their wide use as a means of detecting glycoproteins by western blotting, and isolating glycoproteins and glycopeptides by affinity chromatography. Lectin arrays have the advantage of requiring small amounts of samples, from picogram [182] to microgram quantities [180], under mild conditions that potentially allow the detection of secondary modifications that might be lost under more traditional sample preparation methods. In addition, this approach does not require glycan release or purification prior to analysis, nor does it rely upon costly and complex MS equipment for analysis.

A recent application of lectin microarrays to the study of E. coli surface glycoconjugates demonstrated that the array was able to differentiate between 21 closely related strains [185,186]. This opens the door to rapid carbohydrate-based bacterial fingerprinting $[180,182,185,186]$. Perhaps more importantly, the platform allowed dynamic alterations in the $E$. coli cell surface glycome to be followed and enables the examination of changes in the glycome in response to the host over time. 
While this study highlights the promise of the technology, there are some limitations in its use for bacterial studies. Since whole bacteria were screened, only accessible carbohydrate motifs on the cell surface were visualized, rather than the whole bacterial glycome. However, as the authors acknowledge, it is probable that the surfaceaccessible carbohydrates are those most likely to be involved in immune recognition and cell adhesion $[185,186]$. Perhaps more importantly, the lack of lectins that recognize sugars unique to bacteria is a significant limitation to this technique. The above study used a group of lectins with wellcharacterized binding to mammalian carbohydrate epitopes, which are also found on bacterial pathogens known to express mammalian-like glycans [184,185,187-190]. Lectin microarrays have the advantage of parallel analyses by many lectins simultaneously, but suffer the disadvantage that they are limited by a panel of approximately 60 existing commercially available lectins. These lectins suffer unexpected cross reactivities and often their interaction with bacterial glycans cannot be predicted $[186,191]$. This is in addition to potential problems, such as linking chemistry and the impact of lectin orientation upon binding. In this regard, alternatives to the use of lectins are being sought, including nonprotein carbohydrate binding moieties or short peptides that might function as lectin analogues [192]. As the repertoire of well-characterized bacterial glycanbinding proteins increases, the applications of lectin binding will likely be expanded and applied to screen the surface glycomes of other bacteria.

\section{Role of the C. jejuni glycome in host-pathogen interactions}

Campylobacter jejuni, a Gram-negative intestinal food-borne pathogen, is a major cause of human gastroenteritis and can be associated with the postinfectious neuropathy Guillain-Barré syndrome [193]. Transmission of C. jejuni predominantly occurs through consumption of contaminated meat products, in particular poultry. Genome sequencing provided evidence of an unexpected capacity for this organism to produce a wide range of glycoconjugates, including LOS, CPS and proteins modified with $N$ or $O$-linked sugars [194]. Glycobiologists have focused their efforts on C. jejuni as a toolbox for understanding bacterial glycan biosynthetic pathways. A wealth of information on the role of these glycoconjugates in pathogenesis exists in the literature, which makes C. jejuni a model organism for the discussion of the role of the glycome in host-pathogen interactions.
The outermost glycoconjugate expressed on the cell surface of $C$. jejuni is the CPS, the major serodeterminant of the Penner serotyping scheme [147]. Genomic analysis of C. jejuni isolates indicates a high degree of genetic diversity in the CPS gene clusters, resulting in a large array of CPS structures and modifications being expressed. Bacterial capsules have long been thought to play a protective role against adverse conditions that bacteria might encounter, either within the host or the environment [195]. The C. jejuni CPS is involved in bacterial adherence and colonization of host tissues, protection of the bacterium from dessication and impairment of phagocytosis or complement mediated killing [146]. CPS structures from several different C. jejuni isolates were determined using solution NMR and, as mentioned earlier, HR-MAS NMR. These studies have illustrated the diversity of this cell surface polysaccharide [148-150,196]. Overall, there is still much to be learned about the role of the CPS in C. jejuni, with regards to host interactions.

The outer membrane of $C$. jejuni is composed of a phase-variable LOS, which mimics several human ganglioside structures [197]. This molecular mimicry in some patients is thought to lead to the development of the autoimmune neuropathies Guillain-Barré syndrome and/or Miller Fisher syndrome, caused by the host immune response to LOS ganglioside mimics that have cross-reactivity with host glycolipids [198]. C. jejuni can vary LOS biosynthesis genes through homopolymeric tract variation, which occurs with a relatively high frequency. LOS diversity can vary owing to environmental pressures, allowing the pathogen to adapt and survive in a variety of environments inside and outside the host. Early work on this glycoconjugate supports a role for LOS in cellular adherence and invasion [199]. The application of analytical methods with increased sensitivity has recently allowed a more detailed analysis of the structural diversity of $C$. jejuni LOS. Sialic acid moieties within LOS have been demonstrated to be $O$-acetylated, while heptoses have been observed to harbor $O$-linked glycine modifications $[200,201]$. The biological role of this additional level of LOS glycan diversity remains to be elucidated.

Whole-genome comparisons of 111 strains of C. jejuni, using comparative phylogenomics, demonstrated that isolates from a variety of hosts fell into two clades, defined as livestock and nonlivestock [202]. Of the genes identified as significantly prevalent in either clade, six genes within the $O$-linked flagellin glycosylation biosynthetic 
locus were of particular interest. The strain NCTC11168, found in the livestock clade, has a greater number of putative flagellin-associated biosynthetic genes (50) compared with a sequenced human isolate, 81-176 (26 genes) [33]. This would suggest that livestock-associated strains have the potential to elaborate more complex flagellin modifications in comparison with strains within the nonlivestock clade. However, the structure of the flagellin glycan of livestock strains is not known. The structure of the flagellin glycan from $C$. jejuni $81-176$ is known and is perhaps one of the most well-studied flagellin glycosylation systems. Flagellin structural proteins, FlaA and FlaB, are decorated with the nine-carbon Pse sugar (5,7-diacetamido-3,5,7,9tetradeoxy-L-glycero- $\alpha$-L-manno-nonulosonic acid [Pse5Ac7Ac], as well as an acetamidino form 5-acetamido-7-acetamidino-3,5,7,9-tetradeoxy-L-glycero- $\alpha$-L-manno-nonulosonic acid [Pse5Ac7Am]). Derivatives of these sugars have also been identified in strains of $C$. jejuni and contribute to the structural variability of flagellin glycosylation $[65,66,203,204]$. In C. jejuni 81-176, glycosylation of flagellin is required for flagellar filament biogenesis and bacterial motility and, therefore, has biological significance [33]. The sites of glycan modification of flagellin are predicted to be surface exposed in the fully folded protein, pointing to a role in surface interactions, either with the host cells or other bacterial cells. Autoagglutination (AAG), a known marker of virulence in some bacterial pathogens, including C. jejuni, has been shown to be dependent upon flagellin glycosylation [33]. Studies of defined mutants, deficient in various glycan-associated biosynthetic genes, pointed to a role for both Pse and PseAM in AAG. A further study of a C. jejuni 81-176 PseA mutant, which only modifies flagellin with a single sugar, Pse5Ac7Ac, demonstrated altered AAG compared with wild-type strains. Interestingly, the mutant exhibited alterations in its ability to adhere to a human intestinal epithelial cell line (INT407 cells). During shorter incubation times, only minor defects were observed but, as incubation times were extended, wild-type cells formed microcolonies apparently interacting via flagellar filaments. By contrast, the PseA mutant adhered as single cells, with no visible microcolony formation [33,205]. In an in vivo study using the ferret diarrheal model of disease, the pse $A$ mutant was attenuated relative to the wild-type $C$. jejuni. Although microcolony formation is the first step in the formation of bacterial biofilms, and $C$. jejuni has been shown to form biofilms in vitro [206], there is no evidence that the pathogen forms biofilms in vivo. In this regard, a recent study investigated the role of the $O$-linked glycosylation locus in chicken colonization. In particular, the gene Cj1324 was shown to be involved in the biosynthesis of novel legionaminic acid derivatives on flagellin of $C$. jejuni $11168 \mathrm{H}$. These modifications altered AAG, cell charge and the ability of the mutant strain to colonize chickens [62]. Studies in these animal models point to a role of flagellin, and flagellin glycosylation, in colonization and disease. As with many studies of bacterial glycomes, the details of pathogen glycan interactions with cells of the host have yet to be explained.

As discussed previously, Campylobacter possesses an $N$-linked protein glycosylation system, found within a $16 \mathrm{kB}$ region of the genome $[39,42]$. Addition of the $N$-linked glycan occurs within the perisplasm, with an estimated 150 periplasmic proteins harboring the target sequence for glycosylation (so-called $N$-linked sequon). Several glycoproteins were identified in early work - Cj1594c, PEB3 and Campylobacter glycoprotein A (CgpA). Glycoproteomic analyses by 2D gels showed the glycosylation of both PEB3 and $\operatorname{CgpA}$ to be dependent upon the presence of a functional PglB protein [207]. Since then, an increasing number of $N$-linked glycoproteins have been identified, contributing to the total C. jejuni glycome. These include VirB10, a component of the type IV secretion system, and an inner membrane lipoprotein, $\mathrm{CjA}$ [208].

Discerning the role the $N$-linked heptasaccharide plays in pathogenesis is challenging. The variety of proteins that are modified would suggest that any mutation that disrupts the $p g l$ pathway will have multiple pleiotropic effects. Indeed, disruption of the $p g l$ biosynthetic pathway results in bacteria that have a diminished capacity to adhere to and invade human intestinal cells (INT407) in vitro and a reduced ability to colonize chickens [205,209-211]. Given the number of glycosylated proteins, attributing a direct role for $N$-linked protein glycosylation in colonization and invasion is tenuous at best. Its role may be more general, and may be related to aspects such as protein stability, protection against proteolytic degradation and promoting specific protein-protein interactions [212,213]. This view is supported by the observation that the $N$-glycosylated VirB10 is less stable when not glycosylated [214]. Loss of $N$-glycosylation appears to affect other bacterial processes, such as iron acquisition [215], amino acid transport and biosynthesis and $O$-linked flagellin glycosylation [214]. As mentioned above, these observations 
could be due to secondary effects. Beyond the ambiguous role of the Pgl glycosylation system in bacterial virulence, studies have opened the door towards the use of this pathway in glycoengineering. This is made possible due to the observation that the entire $p g l$ locus can be functionally transferred into E. coli [39] and the fact that the oligosaccharyltransferase $\mathrm{PglB}$ to possess relaxed specificity for the donor glycan [43].

\section{Future perspective}

Despite the growing awareness of the biological significance of bacterial glycoconjugate structures, the study of bacterial glycomics is still in its infancy. Neither the role of bacterial glycans in virulence nor the impact of the bacterial glycome in host-pathogen interactions is clear. Many studies have instead focused upon the eukaryotic host carbohydrates to which many bacterial pathogens bind. As such, there is a pressing need for the adaptation of methods and bioinformatic tools designed for eukaryotic glycomic studies to the study of bacterial glycans. For example, eukaryotic glycan libraries are already being created for use in large-scale studies of carbohydrates. In addition to the traditional, albeit complicated, chemical methods, there are now chemoenzymatic approaches being employed that take advantage of known glycosyltransferases [216]. This sort of technology needs to be adapted for the production of bacterial glycans on a large scale. However, this progress is, and will likely continue to be, hindered by the fact that microbes tend to produce such diverse, unique and unusual sugar modifications.

Although in vitro studies are important for the dissection and study of individual glycans, there will need to be a shift towards increased in vivo work, as the field of bacterial glycomics continues to grow. It is only through the integration of both in vitro and in vivo studies that we will begin to fully understand the biological role and significance of bacterial glycans on a molecular level, particularly with respect to the interplay between pathogens and their hosts. The dynamic nature of the bacterial glycome should also be investigated by future in vivo research, directed at determining how the glycome changes over the course of infection and is impacted by conditions within a host. There is already preliminary evidence that LPS glycoforms of NTHi become shorter and less complex over the course of infection, possibly owing to environmental stresses such as the host's immune response or as a result of depleted nutrients [112]. This may be the most challenging task faced by bacterial glycomics, as it is in proteomics as well, owing to the inherent difficulties associated with studying bacteria grown within a host.

A full understanding of the complex relationship between a pathogen and its host does not lie in a single field of study. Despite its significance, glycomics alone will not provide a complete picture. The potential exists for future systems-level analyses through the incorporation of continued advancements in other fields, such as genomics, proteomics, transcriptomics, lipidomics and metabolomics with the ever-growing field of glycomics. In particular, advancements in genome sequencing technologies will certainly improve understanding of the genetic basis of glycosylation. Furthermore, metabolomic-based studies of lipidlinked intermediates of glycoconjugate molecules will help in the elucidation of their biosynthetic pathways. Only through such multidisciplinary collaboration will a complete understanding of host-pathogen interactions be possible.

As the field of pathogen glycomics continues to grow, there will be significant potential for diagnostic and therapeutic applications. Glycoengineering, in particular, offers a number of medically relevant opportunities based on bacterial glycomic research. This technology has the capacity to produce humanized glycoprotein therapeutics and to alter protein-associated carbohydrates to improve both the activity and half-life of these therapeutic proteins [217]. With advances in glycoengineering, the large-scale production of recombinant glycoconjugate antigens for use in the diagnosis of infection and vaccination against known pathogens will also be possible $[218,219]$. In addition to glycoengineering, inhibitors designed to disrupt the biosynthetic pathways of glycan moieties shown to be involved in pathogen virulence may offer alternative treatment options to antibiotics. This is particularly important given the ever-growing issue of antibiotic resistance.

\section{Acknowledgements}

The authors thank Dr Anne Reid for critical reading of the manuscript.

\section{Financial \& competing interests disclosure}

The authors have no relevant affliations or financial involvement with any organization or entity with a financial interest in or financial conflict with the subject matter or materials discussed in the manuscript. This includes employment, consultancies, honoraria, stock ownership or options, expert testimony, grants or patents received or pending, or royalties.

No writing assistance was utilized in the production of this manuscript. 


\section{Mass spectrometry of the bacterial glycome}

- Mass spectrometry is a pivotal technology in glycomics studies.

\section{Analysis of bacterial glycoproteins}

- There are increasing numbers of reports of bacterial glycosylation in pathogenic bacteria.

- Discovery of bacterial glycoproteins can be challenging due to the diversity of glycans bacteria use to modify proteins

- To date, well-characterized bacterial glycoproteins include pilins, flagellin and other surface-associated proteins of both Gram-negative and Gram-positive bacteria.

- Single proteins, isolated by gel electrophoresis or purification, can be analyzed at the protein or peptide level.

- At the protein level, so-called 'top-down' mass spectrometry studies of intact protein can rapidly characterize bacterial glycan ions.

- At the peptide level, interpretation of individual bacterial glycopeptide tandem mass spectra can be challenging, owing to the diverse range of bacterial glycans produced.

- A combination of mass spectrometry and nuclear magnetic resonance (NMR) over many years has been used to increase our knowledge of the structures of glycans that form the bacterial glycoproteome.

- Reports of methods to specifically isolate bacterial glycopeptides are advancing knowledge of bacterial glycoproteomes.

- Metabolomics studies offer an alternative approach to study $\mathrm{N}$ - or $\mathrm{O}$-linked protein glycosylation biosynthetic pathways.

\section{Application of NMR to bacterial glycomics}

- High-resolution magic angle spinning (HR-MAS) NMR is a powerful tool for the screening of bacterial capsular polysaccharides, and a high-throughput method for identifying genes that assemble or modify bacterial carbohydrates.

- The application of HR-MAS NMR to the analysis of the mycobacterial cell wall provides a valuable tool for studying the biosynthesis of the various carbohydrate components of this highly complex structure.

- Solid-state NMR is one of the only methods to study peptidoglycan tertiary structure, as this insoluble, heterogeneous bacterial polymer is not amenable to classical structrural analytical techniques, such as x-ray crystallography. The ability to study cell wall drug interactions using this technique will provide valuable information towards the development of new or modified antimicrobials.

\section{Probing the bacterial glycome using chemical \& physical methods}

- Lectins (carbohydrate-binding proteins) have been used as tools for glycoprofiling, most recently in microarray formats. This is a high-throughput approach that has the potential to differentiate between bacterial strains, based upon surface carbohydrate profiles

- Lectin microarrays have also been used to characterize the dynamics of the bacterial surface glycome during the course of infection

- The use of lectins in bacterial studies is hindered by a lack of predictable lectin binding to bacterial sugars. Lectins also suffer from sometimes unpredictable cross reactivities.

- Incorporation of sugar analogues into eukaryotic glycans has been achieved and exploited for imaging the glycome. Metabolic labeling of Campylobacter jejuni flagellin glycan was carried out using an azido-labeled pseudaminic acid precursor. This could be exploited in the future for labeling for imaging and other purposes.

\section{Role of the $\boldsymbol{C}$. jejuni glycome in host-pathogen interactions}

- Possibly the best characterized bacterial glycome is that of Campylobacter. This bacterium devotes a considerable proportion of its genome to carbohydrate biosynthesis.

- Studies typically generate deletions of individual glycan-associated biosynthetic genes, and monitor the impact upon glycan structure and bacterial virulence in model systems.

- Components of the $C$. jejuni glycome have a key role in the interaction with the host, including adherence and biofilm formation.

- The molecular targets on host cells with which bacterial glycan interact are largely unknown.

- Rapid progress is leading to an increasing number of studies to determine the role of individual bacterial glycans in interactions with the host.

\section{Bibliography}

Papers of special note have been highlighted as:

- of interest

-" of considerable interest

1. Roseman S: Reflections on glycobiology. J. Biol. Chem. 276(45), 41527-41542 (2001).

2. German JB, Gillies LA, Smilowitz JT, Zivkovic AM, Watkins SM: Lipidomics and lipid profiling in metabolomics. Curr. Opin. Lipidol. 18(1), 66-71 (2007).

3. Dettmer K, Aronov PA, Hammock BD: Mass spectrometry-based metabolomics. Mass Spectrom. Rev. 26(1), 51-78 (2007).
4. Schmidt MA, Riley LW, Benz I: Sweet new world: glycoproteins in bacterial pathogens. Trends Microbiol. 11(12), 554-561 (2003).

- Comprehensive review of bacterial glycoproteins.

5. Lerouge I, Vanderleyden J: $O$-antigen structural variation: mechanisms and possible roles in animal/plant-microbe interactions. FEMS Microbiol. Rev. 26(1), 17-47 (2002).

6. Weintraub A: Immunology of bacterial polysaccharide antigens. Carbohydr. Res. 338(23), 2539-2547 (2003).
7. Ryder C, Byrd M, Wozniak DJ: Role of polysaccharides in Pseudomonas aeruginosa biofilm development. Curr. Opin. Microbiol. 10(6), 644-648 (2007).

8. Messner P: Prokaryotic glycoproteins: unexplored but important. J. Bacteriol. 186(9), 2517-2519 (2004).

9. Logan SM: Flagellar glycosylation - a new component of the motility repertoire? Microbiology 152(Pt 5), 1249-1262 (2006).

- Extensive review of flagellar glycosylation and its role in bacterial motility, and possible interactions with the host. 
10. Laine RA: A calculation of all possible oligosaccharide isomers both branched and linear yields $1.05 \times 10\left({ }^{12}\right)$ structures for a reducing hexasaccharide: the isomer barrier to development of single-method saccharide sequencing or synthesis systems. Glycobiology 4(6), 759-767 (1994).

11. Voisin S, Houliston RS, Kelly J et al.: Identification and characterization of the unique $N$-linked glycan common to the flagellins and S-layer glycoprotein of Methanococcus voltae. J. Biol. Chem. 280(17), 16586-16593 (2005).

12. Twine SM, Paul CJ, Vinogradov E et al.: Flagellar glycosylation in Clostridium botulinum. FEBS J. 275(17), 4428-4444 (2008).

13. Mescher MF, Strominger JL: Structural (shape-maintaining) role of the cell surface glycoprotein of Halobacterium salinarium. Proc. Natl Acad. Sci. USA 73(8), 2687-2691 (1976).

14. Mescher MF, Strominger JL: Purification and characterization of a prokaryotic glucoprotein from the cell envelope of Halobacterium salinarium. J. Biol. Chem. 251(7), 2005-2014 (1976).

15. Szymanski CM, Wren BW: Protein glycosylation in bacterial mucosal pathogens. Nat. Rev. Microbiol. 3(3), 225-237 (2005).

16. Szymanski CM, Yao R, Ewing CP, Trust TJ, Guerry P: Evidence for a system of general protein glycosylation in Campylobacter jejuni. Mol. Microbiol. 32(5), 1022-1030 (1999).

17. Schirm M, Soo EC, Aubry AJ, Austin J, Thibault P, Logan SM: Structural, genetic and functional characterization of the flagellin glycosylation process in Helicobacter pylori. Mol. Microbiol. 48(6), 1579-1592 (2003).

18. Castric P, Cassels FJ, Carlson RW: Structural characterization of the Pseudomonas aeruginosa 1244 pilin glycan. J. Biol. Chem. 276(28), 26479-26485 (2001).

19. Schirm M, Arora SK, Verma A et al:: Structural and genetic characterization of glycosylation of type A flagellin in Pseudomonas aeruginosa. J. Bacteriol. 186(9), 2523-2531 (2004)

20. Banerjee A, Ghosh SK: The role of pilin glycan in neisserial pathogenesis. Mol. Cell. Biochem. 253(1-2), 179-190 (2003).

21. Stimson E, Virji M, Makepeace K et al:: Meningococcal pilin: a glycoprotein substituted with digalactosyl 2,4-diacetamido-2,4,6-trideoxyhexose. Mol. Microbiol. 17(6), 1201-1214 (1995).

22. Lindenthal C, Elsinghorst EA: Identification of a glycoprotein produced by enterotoxigenic Escherichia coli. Infect. Immun. 67(8), 4084-4091 (1999).
23. Benz I, Schmidt MA: Glycosylation with heptose residues mediated by the $A A H$ gene product is essential for adherence of the AIDA-I adhesin. Mol. Microbiol. 40(6), 1403-1413 (2001).

24. Twine SM, Reid CW, Aubry A et al:: Motility and flagellar glycosylation in Clostridium difficile. J. Bacteriol. 191(22), 7050-7062 (2009).

25. Ghosh SK, Zhao J, Philogene MC, Alzaharani A, Rane S, Banerjee A: Pathogenic consequences of Neisseria gonorrhoeae pilin glycan variation. Microbes Infect. 6(7), 693-701 (2004).

26. Ng SY, Chaban B, Jarrell KF: Archaeal flagella, bacterial flagella and type IV pili: a comparison of genes and posttranslational modifications. J. Mol. Microbiol. Biotechnol. 11(3-5), 167-191 (2006).

27. Voisin S, Kus JV, Houliston S et al:: Glycosylation of Pseudomonas aeruginosa strain Pa5196 type IV pilins with mycobacterium-like $\alpha$-1,5-linked D-Araf oligosaccharides. J. Bacteriol. 189(1), 151-159 (2007).

28. Wu H, Zeng M, Fives-Taylor P: The glycan moieties and the $\mathrm{N}$-terminal polypeptide backbone of a fimbria-associated adhesin, Fap1, play distinct roles in the biofilm development of Streptococcus parasanguinis. Infect. Immun. 75(5), 2181-2188 (2007).

29. Zhou M, Wu H: Glycosylation and biogenesis of a family of serine-rich bacterial adhesins. Microbiology 155(Pt 2), 317-327 (2009).

30. Takahashi Y, Sandberg AL, Ruhl S, Muller J, Cisar JO: A specific cell surface antigen of Streptococcus gordonii is associated with bacterial hemagglutination and adhesion to a2-3-linked sialic acid-containing receptors. Infect. Immun. 65(12), 5042-5051 (1997).

31. Plummer C, Wu H, Kerrigan SW, Meade G, Cox D, Ian Douglas CW: A serine-rich glycoprotein of Streptococcus sanguis mediates adhesion to platelets via GPIb. Br. J. Haematol. 129(1), 101-109 (2005).

32. Schaffer C, Messner P: Surface-layer glycoproteins: an example for the diversity of bacterial glycosylation with promising impacts on nanobiotechnology. Glycobiology 14(8), 31R-42R (2004).

33. Guerry P, Ewing CP, Schirm M et al.: Changes in flagellin glycosylation affect Campylobacter autoagglutination and virulence. Mol. Microbiol. 60(2), 299-311 (2006).

34. Schirm M, Kalmokoff M, Aubry A, Thibault P, Sandoz M, Logan SM: Flagellin from Listeria monocytogenes is glycosylated with $\beta$ - $O$-linked $N$-acetylglucosamine. J. Bacteriol. 186(20), 6721-6727 (2004).
35. Grass S, Buscher AZ, Swords WE et al.: The Haemophilus influenzae HMW1 adhesin is glycosylated in a process that requires HMW1C and phosphoglucomutase, an enzyme involved in lipooligosaccharide biosynthesis. Mol. Microbiol. 48(3), 737-751 (2003).

36. Weerapana E, Imperiali B: Asparagine-linked protein glycosylation: from eukaryotic to prokaryotic systems. Glycobiology 16(6), 91R-101R (2006).

37. Kus JV, Kelly J, Tessier L, Harvey H, Cvitkovitch DG, Burrows LL: Modification of Pseudomonas aeruginosa Pa5196 type IV pilins at multiple sites with D-Araf by a novel GT-C family Arabinosyltransferase, TfpW. J. Bacteriol. 190 (22), 7464-7478 (2008).

38. Kus JV, Tullis E, Cvitkovitch DG, Burrows LL: Significant differences in type IV pilin allele distribution among Pseudomonas aeruginosa isolates from cystic fibrosis (CF) versus non-CF patients. Microbiology 150(Pt 5), 1315-1326 (2004).

39. Young NM, Brisson JR, Kelly J et al.: Structure of the $N$-linked glycan present on multiple glycoproteins in the Gram-negative bacterium, Campylobacter jejuni. J. Biol. Chem. 277(45), 42530-42539 (2002).

40. Kelly J, Jarrell H, Millar L et al:: Biosynthesis of the $\mathrm{N}$-linked glycan in Campylobacter jejuni and addition onto protein through block transfer. J. Bacteriol. 188(7), 2427-2434 (2006).

41. Kowarik M, Young NM, Numao S et al:: Definition of the bacterial $N$-glycosylation site consensus sequence. EMBO J.25(9), 1957-1966 (2006).

42. Wacker M, Linton D, Hitchen PG et al.: $N$-linked glycosylation in Campylobacter jejuni and its functional transfer into $E$. coli. Science 298(5599), 1790-1793 (2002).

- The cloning of Campylobacter jejuni $N$-linked glycosylation cassette in Escherichia coli opened up the possibility of engineering recombinant glycan structures.

43. Wacker M, Feldman MF, Callewaert $\mathrm{N}$ et al.: Substrate specificity of bacterial oligosaccharyltransferase suggests a common transfer mechanism for the bacterial and eukaryotic systems. Proc. Natl Acad. Sci. USA 103(18), 7088-7093 (2006).

44. Hounsell EF, Davies MJ, Smith KD: $O$-linked oligosaccharide profiling by HPAEC-PAD. In: Protein Protocols Handbook. Walker J (Ed.). Humana Press, NY, USA, 651-652 (1996)

45. Harvey DJ: Analysis of carbohydrates and glycoconjugates by matrix-assisted laser desorption/ionization mass spectrometry: an update for 2003-2004. Mass Spectrom. Rev. 28(2), 273-361 (2009). 
46. Harvey DJ, Dwek RA, Rudd PM: Determining the structure of glycan moieties by mass spectrometry. Curr. Protoc. Protein Sci. Chapter 12, Unit 12.7 (2006).

47. Harvey DJ: Analysis of carbohydrates and glycoconjugates by matrix-assisted laser desorption/ionization mass spectrometry: an update covering the period 2001-2002. Mass Spectrom. Rev. 27(2), 125-201 (2008).

48. Harvey DJ: Proteomic analysis of glycosylation: structural determination of $\mathrm{N}$ - and $O$-linked glycans by mass spectrometry. Expert Rev. Proteomics 2(1), 87-101 (2005).

49. Ortner K, Sivanandam VN, Buchberger W, Muller N: Analysis of glycans in glycoproteins by diffusion-ordered nuclear magnetic resonance spectroscopy. Anal. Bioanal. Chem. 388(1), 173-177 (2007).

50. Wilson NL, Schulz BL, Karlsson NG, Packer NH: Sequential analysis of $N$ - and $O$-linked glycosylation of 2D-PAGE separated glycoproteins. J. Proteome Res. 1(6), 521-529 (2002).

51. Guttman A: High-resolution carbohydrate profiling by capillary gel electrophoresis. Nature 380 (6573), 461-462 (1996).

52. Koller A, Khandurina J, Li J, Kreps J, Schieltz D, Guttman A: Analysis of high-mannose-type oligosaccharides by microliquid chromatography-mass spectrometry and capillary electrophoresis. Electrophoresis 25(13), 2003-2009 (2004).

53. Olajos M, Hajos P, Bonn GK, Guttman A: Sample preparation for the analysis of complex carbohydrates by multicapillary gel electrophoresis with light-emitting diode induced fluorescence detection. Anal. Chem. 80(11), 4241-4246 (2008).

54. Hitchen PG, Dell A: Bacterial glycoproteomics. Microbiology 152(Pt 6), 1575-1580 (2006).

55. Twine SM, Tessier L, Kelly JF: Characterisation of glycoproteins. In: Functional Glycomics, Methods and Protocols. Li J (Ed.). Humana Press, NY, USA (2009).

56. Schirm M, Schoenhofen IC, Logan SM, Waldron KC, Thibault P: Identification of unusual bacterial glycosylation by tandem mass spectrometry analyses of intact proteins. Anal. Chem. 77(23), 7774-7782 (2005).

- Description of 'top-down' mass spectrometry analysis of intact bacterial glycoproteins, including identification of novel protein-associated glycans. Method was also applied to detect flagellin from a mixture of intact proteins.

57. Carter AT, Paul CJ, Mason DR et al.: Independent evolution of neurotoxin and flagellar genetic loci in proteolytic Clostridium botulinum. BMC Genomics 10, 115 (2009).
58. Balonova L, Hernychova L, Bilkova Z: Bioanalytical tools for the discovery of eukaryotic glycoproteins applied to the analysis of bacterial glycoproteins. Expert Rev. Proteomics 6(1), 75-85 (2009).

- Review of modern bioanalytical techniques applied for bacterial glycoprotein detection and enrichment from complex samples.

59. Ding W, Nothaft H, Szymanski CM, Kelly J: Identification and quantification of glycoproteins using ion-pairing normal-phase LC and MS. Mol. Cell. Proteomics 8, 2170-2185 (2009).

-. High-performance liquid chromatographybased technique was developed to specifically enrich glycopeptides from a periplasmic extract of $C$. jejuni and aided in the identification of previously undetermined $N$-linked glycoproteins.

60. Ding W, Hill JJ, Kelly J: Selective enrichment of glycopeptides from glycoprotein digests using ion-pairing normal-phase liquid chromatography. Anal. Chem. 79(23), 8891-8899 (2007).

61. Liu X, McNally DJ, Nothaft $\mathrm{H}$, Szymanski CM, Brisson JR, Li J: Mass spectrometry-based glycomics strategy for exploring $N$-linked glycosylation in eukaryotes and bacteria. Anal. Chem. 78(17), 6081-6087 (2006).

62. Howard SL, Jagannathan A, Soo EC et al.: Campylobacter jejuni glycosylation island important in cell charge, legionaminic acid biosynthesis, and colonization of chickens. Infect. Immun. 77(6), 2544-2556 (2009).

63. Hui JP, Yang J, Thorson JS, Soo EC: Selective detection of sugar phosphates by capillary electrophoresis/mass spectrometry and its application to an engineered $E$. coli host. Chembiochem 8(10), 1180-1188 (2007).

64. Logan SM, Hui JP, Vinogradov E et al: Identification of novel carbohydrate modifications on Campylobacter jejuni 11168 flagellin using metabolomics-based approaches. FEBS J. 276(4), 1014-1023 (2009).

- Advanced targeted metabolomics methodologies used to identify novel glycans.

65. McNally DJ, Hui JP, Aubry AJ et al: Functional characterization of the flagellar glycosylation locus in Campylobacter jejuni 81-176 using a focused metabolomics approach. J. Biol. Chem. 281(27), 18489-18498 (2006).

66. McNally DJ, Aubry AJ, Hui JP et al.: Targeted metabolomics analysis of Campylobacter coli VC167 reveals legionaminic acid derivatives as novel flagellar glycans. J. Biol. Chem. 282(19), 14463-14475 (2007).
67. Soo EC, Aubry AJ, Logan SM et al.: Selective detection and identification of sugar nucleotides by $\mathrm{CE}$-electrospray-MS and its application to bacterial metabolomics. Anal. Chem. 76(3), 619-626 (2004).

68. Bino RJ, Hall RD, Fiehn O et al.: Potential of metabolomics as a functional genomics tool. Trends Plant Sci. 9(9), 418-425 (2004).

69. Mashego MR, Wu L, van Dam JC et al: : MIRACLE: mass isotopomer ratio analysis of U-13C-labeled extracts. A new method for accurate quantification of changes in concentrations of intracellular metabolites. Biotechnol. Bioeng. 85(6), 620-628 (2004).

70. Mashego MR, Rumbold K, De MM, Vandamme E, Soetaert W, Heijnen JJ: Microbial metabolomics: past, present and future methodologies. Biotechnol. Lett. 29(1), 1-16 (2007).

71. Kussak A, Weintraub A: Quadrupole ion-trap mass spectrometry to locate fatty acids on lipid A from Gram-negative bacteria. Anal. Biochem. 307(1), 131-137 (2002).

72. Murphy RC, Raetz CRH, Reynolds CM, Barkley RM: Mass spectrometry advances in lipidomics: collision-induced decomposition of $\mathrm{Kdo}_{2}$-lipid A. Prostaglandins Other Lipid Mediat. 77, 131-140 (2005).

73. Gao N, Lehrman MA: Analyses of dolichol pyrophosphate-linked oligosaccharides in cell cultures and tissues by fluorophore-assisted carbohydrate electrophoresis. Glycobiology 12(5), 353-360 (2002).

74. Barreteau H, Magnet S, El GM et al.: Quantitative high-performance liquid chromatography analysis of the pool levels of undecaprenyl phosphate and its derivatives in bacterial membranes. J. Chromatogr. B Analyt. Technol. Biomed. Life Sci. 877(3), 213-220 (2009).

75. Guan Z, Breazeale SD, Raetz CR: Extraction and identification by mass spectrometry of undecaprenyl diphosphate-MurNAcpentapeptide-GlcNAc from Escherichia coli. Anal. Biochem. 345(2), 336-339 (2005).

76. Trent MS, Ribeiro AA, Doerrler WT, Lin S, Cotter RJ, Raetz CR: Accumulation of a polyisoprene-linked amino sugar in polymyxin-resistant Salmonella Typhimurium and Escherichia coli: structural characterization and transfer to lipid $\mathrm{A}$ in the periplasm. J. Biol. Chem. 276(46), 43132-43144 (2001)

77. Trent MS, Ribeiro AA, Lin S, Cotter RJ, Raetz CR: An inner membrane enzyme in Salmonella and Escherichia coli that transfers 4-amino-4-deoxy-L-arabinose to lipid A: induction on polymyxin-resistant mutants and role of a novel lipid-linked donor. J. Biol. Chem. 276(46), 43122-43131 (2001). 
78. Wang X, Ribeiro AA, Guan Z, Raetz CR: Identification of undecaprenyl phosphate- $\beta$ D-galactosamine in Francisella novicida and its function in lipid A modification. Biochemistry 48(6), 1162-1172 (2009).

79. Reid CW, Stupak J, Chen MM, Imperiali B, Li J, Szymanski CM: Affinity-capture tandem mass spectrometric characterization of polyprenyl-linked oligosaccharides: tool to study protein $\mathrm{N}$-glycosylation pathways. Anal. Chem. 80(14), 5468-5475 (2008).

- Uses a lectin affinity method to capture and enrich for low levels of lipid-linked oligosaccharide intermediates of the C. jejuni Pgl pathways with minimal sample manipulation.

80. Karaoglu D, Kelleher DJ, Gilmore R: Allosteric regulation provides a molecular mechanism for preferential utilization of the fully assembled dolichol-linked oligosaccharide by the yeast oligosaccharyltransferase. Biochemistry 40 (40), 12193-12206 (2001).

81. Lehrman MA: Teaching dolichol-linked oligosaccharides more tricks with alternatives to metabolic radiolabeling. Glycobiology 17(8), 75R-85R (2007).

82. Quigley WW, Dovichi NJ: Capillary electrophoresis for the analysis of biopolymers. Anal. Chem. 76(16), 4645-4658 (2004).

83. Li J, Purves RW, Richards JC: Coupling capillary electrophoresis and high-field asymmetric waveform ion mobility spectrometry mass spectrometry for the analysis of complex lipopolysaccharides. Anal. Chem. 76(16), 4676-4683 (2004).

84. Li J, Cox AD, Hood DW, Schweda EK, Moxon ER, Richards JC: Electrophoretic and mass spectrometric strategies for profiling bacterial lipopolysaccharides. Mol. Biosyst. 1(1), 46-52 (2005).

85. Li J, Martin A, Cox AD, Moxon ER, Richards JC, Thibault P: Mapping bacterial glycolipid complexity using capillary electrophoresis and electrospray mass spectrometry. Methods Enzymol. 405, 369-397 (2005).

86. Thibault P, Richards JC: Applications of combined capillary electrophoresiselectrospray mass spectrometry in the characterization of short-chain lipopolysaccharides. Haemophilus influenzae. Methods Mol. Biol. 145, 327-344 (2000).

87. Thibault P, Martin A, Gilbert M, Wakarchuk WW, Richards JC: Analysis of bacterial glycolipids by capillary electrophoresis-electrospray mass spectrometry: Haemophilus influenzae and Neisseria meningitidis lipopolysaccharides. Methods Mol. Biol. 213, 241-259 (2003).
88. Li J, Cox AD, Hood D, Moxon ER, Richards JC: Application of capillary electrophoresiselectrospray-mass spectrometry to the separation and characterization of isomeric lipopolysaccharides of Neisseria meningitidis. Electrophoresis 25(13), 2017-2025 (2004).

89. St Michael F, Li J, Cox AD: Structural analysis of the core oligosaccharide from Pasteurella multocida strain X73. Carbohydr. Res. 340(6), 1253-1257 (2005).

90. St Michael F, Li J, Vinogradov E, Larocque S, Harper M, Cox AD: Structural analysis of the lipopolysaccharide of Pasteurella multocida strain VP161: identification of both Kdo-P and Kdo-Kdo species in the lipopolysaccharide. Carbohydr. Res. 340 (1), 59-68 (2005).

91. St Michael F, Vinogradov E, Li J, Cox AD: Structural analysis of the lipopolysaccharide from Pasteurella multocida genome strain Pm70 and identification of the putative lipopolysaccharide glycosyltransferases. Glycobiology 15(4), 323-333 (2005).

92. Altman E, Smirnova N, Li J, Aubry A, Logan SM: Occurrence of a nontypable Helicobacter pylori strain lacking Lewis blood group $\mathrm{O}$ antigens and DD-heptoglycan: evidence for the role of the core $\alpha 1,6$-glucan chain in colonization. Glycobiology 13(11), 777-783 (2003).

93. Logan SM, Altman E, Mykytczuk O et al.: Novel biosynthetic functions of lipopolysaccharide rfaJ homologs from Helicobacter pylori. Glycobiology 15(7), 721-733 (2005).

94. Godschalk PC, Kuijf ML, Li J et al. Structural characterization of Campylobacter jejuni lipooligosaccharide outer cores associated with Guillain-Barre and Miller Fisher syndromes. Infect. Immun. 75(3), 1245-1254 (2007).

95. Gilbert M, Godschalk PC, Karwaski MF et al.: Evidence for acquisition of the lipooligosaccharide biosynthesis locus in Campylobacter jejuni $\mathrm{GB} 11$, a strain isolated from a patient with Guillain-Barre syndrome, by horizontal exchange. Infect. Immun. 72(2), 1162-1165 (2004).

96. Szymanski CM, Michael FS, Jarrell HC et al: Detection of conserved $N$-linked glycans and phase-variable lipooligosaccharides and capsules from Campylobacter cells by mass spectrometry and high resolution magic angle spinning NMR spectroscopy. J. Biol. Chem. 278(27), 24509-24520 (2003).

- Study of capsular polysaccharide from intact bacterial cells, using high-resolution magic angle spinning. Shows the potential for this method in serotyping and preliminary sugar analyses. Protein $N$-linked glycans were also detected using this method.
97. Wang Z, Vinogradov E, Li J, Lund V, Altman E: Structural characterization of the lipopolysaccharide $O$-antigen from atypical isolate of Vibrio anguillarum strain 1282. Carbohydr. Res. 344(11), 1371-1375 (2009).

98. Wang Z, Li J, Vinogradov E, Altman E: Structural studies of the core region of Aeromonas salmonicida subsp. salmonicida lipopolysaccharide. Carbohydr. Res. 341(1), 109-117 (2006)

99. Li J, Thibault P, Martin A, Richards JC, Wakarchuk WW, van der Wilp W: Development of an on-line preconcentration method for the analysis of pathogenic lipopolysaccharides using capillary electrophoresis-electrospray mass spectrometry. Application to small colony isolates. J. Chromatogr. A 817(1-2), 325-336 (1998).

100. Wang Z, Liu X, Garduno E, Garduno RA, Li J, Altman E: Application of an immunoaffinity-based preconcentration method for mass spectrometric analysis of the $O$-chain polysaccharide of Aeromonas salmonicida from in vitro- and in vivo-grown cells. FEMS Microbiol. Lett. 295(2), 148-155 (2009).

101. Wang Z, Vinogradov E, Larocque $S$, Harrison BA, Li J, Altman E: Structural and serological characterization of the $O$-chain polysaccharide of Aeromonas salmonicida strains A449, 80204 and 80204-80201. Carbohydr. Res. 340(4), 693-700 (2005).

102. Pichichero ME: Pathogen shifts and changing cure rates for otitis media and tonsillopharyngitis. Clin. Pediatr. (Phila.) 45(6), 493-502 (2006).

103. Cox AD, Masoud H, Thibault P et al: Structural analysis of the lipopolysaccharide from the nontypable Haemophilus influenzae strain SB 33. Eur. J. Biochem. 268(20), 5278-5286 (2001).

104. Mansson M, Hood DW, Moxon ER, Schweda EK: Structural characterization of a novel branching pattern in the lipopolysaccharide from nontypeable Haemophilus influenzae. Eur. J. Biochem. 270(14), 2979-2991 (2003).

105. Mansson M, Hood DW, Moxon ER, Schweda EK: Structural diversity in lipopolysaccharide expression in nontypeable Haemophilus influenzae. Identification of L-glycerol-D-manno-heptose in the outer-core region in three clinical isolates. Eur. J. Biochem. 270(4), 610-624 (2003).

106. Mansson M, Hood DW, Li J, Richards JC, Moxon ER, Schweda EK: Structural analysis of the lipopolysaccharide from nontypeable Haemophilus influenzae strain 1003. Eur.J. Biochem. 269(3), 808-818 (2002). 
107. Mansson M, Bauer SH, Hood DW, Richards JC, Moxon ER, Schweda EK: A new structural type for Haemophilus influenzae lipopolysaccharide. Structural analysis of the lipopolysaccharide from nontypeable Haemophilus influenzae strain 486. Eur. J. Biochem. 268(7), 2148-2159 (2001).

108. Yildirim HH, Li J, Richards JC, Hood DW, Moxon ER, Schweda EK: Complex $O$-acetylation in non-typeable Haemophilus influenzae lipopolysaccharide: evidence for a novel site of $O$-acetylation. Carbohydr. Res. 340 (17), 2598-2611 (2005).

109. Yildirim HH, Li J, Richards JC, Hood DW, Moxon ER, Schweda EK: An alternate pattern for globoside oligosaccharide expression in Haemophilus influenzae lipopolysaccharide: structural diversity in nontypeable strain 1124. Biochemistry 44(13), 5207-5224 (2005).

110. Bouchet V, Hood DW, Li J et al.: Host-derived sialic acid is incorporated into Haemophilus influenzae lipopolysaccharide and is a major virulence factor in experimental otitis media. Proc. Natl Acad. Sci. USA 100(15), 8898-8903 (2003).

111. Figueira MA, Ram S, Goldstein R, Hood DW, Moxon ER, Pelton SI: Role of complement in defense of the middle ear revealed by restoring the virulence of nontypeable Haemophilus influenzae siaB mutants. Infect. Immun. 75(1), 325-333 (2007).

112. Erwin AL, Smith AL: Nontypeable Haemophilus influenzae: understanding virulence and commensal behavior. Trends Microbiol. 15(8), 355-362 (2007).

113. Greiner LL, Watanabe H, Phillips NJ et al.: Nontypeable Haemophilus influenzae strain 2019 produces a biofilm containing $N$-acetylneuraminic acid that may mimic sialylated $O$-linked glycans. Infect. Immun. 72(7), 4249-4260 (2004).

114. Jurcisek J, Greiner L, Watanabe H, Zaleski A, Apicella MA, Bakaletz LO: Role of sialic acid and complex carbohydrate biosynthesis in biofilm formation by nontypeable Haemophilus influenzae in the chinchilla middle ear. Infect. Immun. 73(6), 3210-3218 (2005).

115. Lundstrom SL, Li J, Mansson M et al.: Application of capillary electrophoresis mass spectrometry and liquid chromatography multiple-step tandem electrospray mass spectrometry to profile glycoform expression during Haemophilus influenzae pathogenesis in the chinchilla model of experimental otitis media. Infect. Immun. 76(7), 3255-3267 (2008).
116. Clowers BH, Hill HH Jr: Mass analysis of mobility-selected ion populations using dual gate, ion mobility, quadrupole ion trap mass spectrometry. Anal. Chem. 77(18), 5877-5885 (2005).

117. Clowers BH, Dwivedi P, Steiner WE, Hill HH Jr, Bendiak B: Separation of sodiated isobaric disaccharides and trisaccharides using electrospray ionizationatmospheric pressure ion mobility-time of flight mass spectrometry. J. Am. Soc. Mass Spectrom. 16(5), 660-669 (2005).

118. Tang K, Li F, Shvartsburg AA, Strittmatter EF, Smith RD: Two-dimensional gas-phase separations coupled to mass spectrometry for analysis of complex mixtures. Anal. Chem. 77(19), 6381-6388 (2005).

119. Venne K, Bonneil E, Eng K, Thibault P: Improvement in peptide detection for proteomics analyses using NanoLC-MS and high-field asymmetry waveform ion mobility mass spectrometry. Anal. Chem. 77(7), 2176-2186 (2005)

120. Goldberg D, Sutton-Smith M, Paulson J, Dell A: Automatic annotation of matrixassisted laser desorption/ionization $N$-glycan spectra. Proteomics 5(4), 865-875 (2005).

121. Tang H, Mechref $Y$, Novotny MV: Automated interpretation of MS/MS spectra of oligosaccharides. Bioinformatics 21(Suppl. 1), i431-i439 (2005).

122. Irungu J, Go EP, Dalpathado DS, Desaire H: Simplification of mass spectral analysis of acidic glycopeptides using GlycoPep ID. Anal. Chem. 79(8), 3065-3074 (2007).

123. Cooper CA, Gasteiger E, Packer NH: GlycoMod - a software tool for determining glycosylation compositions from mass spectrometric data. Proteomics 1(2), 340-349 (2001).

124. Maass K, Ranzinger R, Geyer H, von der Lieth CW, Geyer R: 'Glycopeakfinder' - de novo composition analysis of glycoconjugates. Proteomics 7(24), 4435-4444 (2007).

125. Hashimoto K, Goto S, Kawano S et al.: KEGG as a glycome informatics resource. Glycobiology 16(5), 63R-70R (2006).

126. von der Lieth CW, Lutteke T, Frank M: The role of informatics in glycobiology research with special emphasis on automatic interpretation of MS spectra. Biochim. Biophys. Acta 1760(4), 568-577 (2006).

127. Raman R, Venkataraman M, Ramakrishnan S, Lang W, Raguram S, Sasisekharan R: Advancing glycomics: implementation strategies at the consortium for functional glycomics. Glycobiology 16(5), 82R-90R (2006).
128. Aoki-Kinoshita KF: An introduction to bioinformatics for glycomics research. PLoS Comput. Biol. 4(5), e1000075 (2008).

129. Eakin RT, Morgan LO, Gregg CT, Matwyoff NA: Carbon-13 nuclear magnetic resonance spectroscopy of living cells and their metabolism of a specifically labeled ${ }^{13} \mathrm{C}$ substrate. FEBS Lett. 28, 259-264 (1972).

130. Bergstrom N, Nair GB, Weintraub A, Jansson PE: Structure of the $O$-polysaccharide from the lipopolysaccharide from Vibrio cholerae O6. Carbohydr. Res. 337(9), 813-817 (2002).

131. Beynon LM, Richards JC, Perry MB: The structure of the lipopolysaccharide $O$ antigen from Yersinia ruckeri serotype 01 . Carbohydr. Res. 256(2), 303-317 (1994).

132. Beynon LM, Moreau M, Richards JC, Perry MB: Structure of the $O$-antigen of Actinobacillus pleuropneumoniae serotype 7 lipopolysaccharide. Carbohydr. Res. 209, 225-238 (1991).

133. Brooks BW, Perry MB, Lutze-Wallace CL, MacLean LL: Structural characterization and serological specificities of lipopolysaccharides from Salmonella enterica serovar Gallinarum biovar Pullorum standard, intermediate and variant antigenic type strains. Vet. Microbiol. 126(4), 334-344 (2008).

134. Bystrova OV, Knirel YA, Lindner B et al.: Structures of the core oligosaccharide and $\mathrm{O}$-units in the R-and SR-type lipopolysaccharides of reference strains of Pseudomonas aeruginosa $O$-serogroups. FEMS Immunol. Med. Microbiol. 46(1), 85-99 (2006).

135. Vinogradov E, Perry MB: Characterisation of the core part of the lipopolysaccharide $O$-antigen of Francisella novicida (U112). Carbohydr. Res. 339(9), 1643-1648 (2004).

136. Vinogradov E, Frirdich E, MacLean LL et al:: Structures of lipopolysaccharides from Klebsiella pneumoniae. Eluicidation of the structure of the linkage region between core and polysaccharide $O$ chain and identification of the residues at the non-reducing termini of the $O$ chains. J. Biol. Chem. 277(28), 25070-25081 (2002).

137. Vinogradov E: The structure of the core- $O$-chain linkage region of the lipopolysaccharide from Bordetella binzii. Carbohydr. Res. 342(3-4), 638-642 (2007).

138. Vinogradov E, Kubler-Kielb J, Korenevsky A: The structure of the carbohydrate backbone of the LPS from Shewanella spp. MR-4. Carbohydr. Res. 343(15), 2701-2705 (2008). 
139. Griffin JL, Bollard M, Nicholson JK, Bhakoo K: Spectral profiles of cultured neuronal and glial cells derived from HRMAS ${ }^{1}$ H NMR spectroscopy. NMR Biomed. 15, 375-384 (2002).

140. Kurhanewicz J, Swanson MG, Nelson SJ, Vineron DB: Combined magnetic resonance imaging and spectroscopic imaging approach to molecular imaging of prostate cancer. J. Magn. Reson. Imaging 16, 451-463 (2002).

141. Cheng LL, Ma MJ, Becerra L et al:: Quantitative neuropathology by high resolution magic angle spinning proton magnetic resonance spectroscopy. Proc. Natl Acad. Sci. USA 94(12), 6408-6413 (1997).

142. Weybright P, Millis K, Campbell N, Cory DG, Singer S: Gradient, highresolution, magic angle spinning ${ }^{1} \mathrm{H}$ nuclear magnetic resonance spectroscopy of intact cells. Magn. Reson. Med. 39(3), 337-345 (1998).

143. Gudlavalleti SK, Szymanski CM, Jarrell HC, Stephens DS: In vivo determination of Neisseria meningitidis serogroup A capsular polysaccharide by whole cell high-resolution magic angle spinning NMR spectroscopy. Carbohydr. Res. 341(4), 557-562 (2006).

144. Lee REB, Li W, Chatterjee D, Lee RE: Rapid structural characterization of the arabinogalactan and lipoarabinomannan in live mycobacterial cells using 2D and 3D HR-MAS NMR: structural changes in the arabinan due to ethambutol treatment and gene mutation are observed. Glycobiology 15(2), 139-151 (2005).

- An interesting application of multidimensional high-resolution magic angle spinning whole-cell nuclear magnetic resonance to the study of the mycobacterial cell wall.

145. Karlyshev AV, Champion OL, Churcher C et al:: Analysis of Campylobacter jejuni capsular loci reveals multiple mechanisms for the generation of structural diversity and the ability to form complex heptoses. Mol. Microbiol. 55(1), 90-103 (2005).

146. Bacon DJ, Szymanski CM, Burr DH, Silver RP, Alm RA, Guerry P: A phasevariable capsule is involved in virulence of Campylobacter jejuni 81-176. Mol. Microbiol. 40(3), 769-777 (2001).

147. Karlyshev AV, Linton D, Gregson NA, Lastovica AJ, Wren BW: Genetic and biochemical evidence of a Campylobacter jejuni capsular polysaccharide that accounts for Penner serotype specificity. Mol. Microbiol. 35(3), 529-541 (2000).

148. McNally DJ, Jarrell HC, Li J et al:: The HS:1 serostrain of Campylobacter jejuni has a complex teichoic acid-like capsular polysaccharide with nonstoichiometric fructofuranose branches and $O$-methyl phosphoramidate groups. FEBS J. 272(17), 4407-4422 (2005).

149. McNally DJ, Jarrell HC, Khieu NH et al.: The HS:19 serostrain of Campylobacter jejuni has a hyaluronic acid-type capsular polysaccharide with a nonstoichiometric sorbose branch and $O$-methyl phosphoramidate group. FEBS J. 273(17), 3975-3989 (2006).

150. McNally DJ, Lamoureux MP, Karlyshev AV et al: Commonality and biosynthesis of the $O$-methyl phosphoramidate capsule modification in Campylobacter jejuni. J. Biol. Chem. 282(39), 28566-28576 (2007).

- Illustrates the usefulness of high-resolution magic angle spinning nuclear magnetic resonance as a high-throughput screen for surface polysaccharide biosynthetic genes and ex vivo analysis of $C$. jejuni in cecal contents of a colonized chick.

151. Li W, Lee REB, Lee RE, Li J: Methods for acquisition and assignment of multidimensional high-resolution magic angle spinning NMR of whole cell bacteria. Anal. Chem. 77, 5785-5792 (2005).

152. Parsons LM, Salfinger M, Clobridge A et al.: Phenotypic and molecular characterization of Mycobacterium tuberculosis isolates resistant to both isoniazid and ethambutol. Antimicrob. Agents Chemother. 49(6), 2218-2225 (2005).

153. Reid CW, Brewer D, Clarke AJ: Substrate binding affinity of Pseudomonas aeruginosa membrane-bound lytic transglycosylase B by hydrogen-deuterium exchange MALDI MS. Biochemistry 43(35), 11275-11282 (2004).

154. Reid CW, Blackburn NT, Clarke AJ: Role of arginine residues in the active site of the membrane-bound lytic transglycosylase B from Pseudomonas aeruginosa. Biochemistry 45(7), 2129-2138 (2006).

155. Tong G, Pan Y, Dong H, Pryor R, Wilson GE, Schaefer J: Structure and dynamics of pentaglycyl bridges in the cell walls of Staphylococcus aureus by $13 \mathrm{C}-15 \mathrm{~N}$ REDOR NMR. Biochemistry 36(32), 9859-9866 (1997).

156. Cegelski L, Steuber D, Mehta AK, Kulp DW, Axelsen PH, Schaefer J: Conformational and quantitative characterization of oritavancinpeptidoglycan complexes in whole cells of Staphylococcus aureus by in vivo ${ }^{13} \mathrm{C}$ and ${ }^{15} \mathrm{~N}$ labeling. J. Mol. Biol. 357, 1253-1262 (2006).

157. Kim SJ, Jones RC, Cha CJ, Kweon O, Edmondson RD, Cerniglia CE: Identification of proteins induced by polycyclic aromatic hydrocarbon in Mycobacterium vanbaalenii PYR-1 using two-dimensional polyacrylamide gel electrophoresis and de novo sequencing methods. Proteomics 4(12), 3899-3908 (2004).

158. Kim SJ, Cegelski L, Preobrazhenskaya M, Schaefer J: Structures of Staphylococcus aureus cell-wall complexes with vancomycin, eremomycin, and chloroeremomycin derivatives by $13 \mathrm{C}\{19 \mathrm{~F}\}$ and $15 \mathrm{~N}\{19 \mathrm{~F}\}$ rotational-echo double resonance. Biochemistry 45(16), 5235-5250 (2006).

159. Patti GJ, Chen J, Schaefer J, Gross ML: Characterization of structural variations in the peptidoglycan of vancomycin-susceptible Enterococcus faecium: understanding glycopeptide-antibiotic binding sites using mass spectrometry. J. Am. Soc. Mass Spectrom. 19(10), 1467-1475 (2008).

160. Patti GJ, Kim SJ, Schaefer J: Characterization of the peptidoglycan of vancomycinsusceptible Enterococcus faecium. Biochemistry 47(32), 8378-8385 (2008).

161. Sharif S, Kim SJ, Labischinski H, Schaefer J: Characterization of peptidoglycan in Fem-deletion mutants of methicillin-resistant Staphylococcus aureus by solid-state NMR. Biochemistry 48, 3100-3108 (2009).

162. Boniface A, Parquet C, Arthur M, Mengin-Lecreulx D, Blanot D: The elucidation of the structure of Thermotoga maritima peptidoglycan reveals two novel types of cross-link. J. Biol. Chem. 284(33), 21856-21862 (2009).

163. Sharif S, Singh M, Kim SJ, Schaefer J: Staphylococcus aureus peptidoglycan tertiary structure from carbon-13 diffusion. J. Am. Chem. Soc. 131, 7023-7030 (2009).

- Provides insight into the Gram-positive cell wall tertiary structure. Demonstrates that solid-state nuclear magnetic resonance can be a very powerful tool in understanding peptidoglycan dynamics.

164. Meroueh SO, Bencze KZ, Hesek D et al: Three-dimensional structure of the bacterial cell wall peptidoglycan. Proc. Natl Acad. Sci. USA 103(12), 4404-4409 (2006).

165. Perez-Dorado I, Campillo NE, Monterroso B et al:: Elucidation of the molecular recognition of bacterial cell wall by modular pneumococcal phage endolysin CPL-1. J. Biol. Chem. 282(34), 24990-24999 (2007).

166. Kim SJ, Matsuoka S, Patti GJ, Schaefer J: Vancomycin derivative with damaged D-Ala-D-Ala binding cleft binds to crosslinked peptidoglycan in the cell wall of Staphylococcus aureus. Biochemistry 47, 3822-3831 (2009).

167. Kim SJ, Cegelski L, Stueber D et al.: Oritavancin exhibits dual mode of action to inhibit cell-wall biosynthesis in Staphylococcus aureus. J. Mol. Biol. 377(1), 281-293 (2008). 
168. Kim SJ, Cegelski L, Studelska DR, O'Connor RD, Mehta AK, Schaefer J: Rotational-echo double resonance characterization of vancomycin binding sites in Staphylococcus aureus. Biochemistry 41(22), 6967-6977 (2002).

169. Agard NJ, Bertozzi CR: Chemical approaches to perturb, profile, and perceive glycans. Acc. Chem. Res. 42(6), 788-797 (2009).

170. Bertozzi CR, Kiessling LL: Chemical glycobiology. Science 291(5512), 2357-2364 (2001).

171. Chang PV, Chen X, Smyrniotis C et al.: Metabolic labeling of sialic acids in living animals with alkynyl sugars. Angew. Chem. Int. Ed. Engl. 48(22), 4030-4033 (2009).

172. Zeng Y, Ramya TN, Dirksen A, Dawson PE, Paulson JC: High-efficiency labeling of sialylated glycoproteins on living cells. Nat. Methods 6(3), 207-209 (2009).

173. Chang PV, Prescher JA, Hangauer MJ, Bertozzi CR: Imaging cell surface glycans with bioorthogonal chemical reporters. J. Am. Chem. Soc. 129(27), 8400-8401 (2007).

174. Gahmberg CG, Andersson LC: Selective radioactive labeling of cell surface sialoglycoproteins by periodate-tritiated borohydride. J. Biol. Chem. 252(16), 5888-5894 (1977).

175. Dirksen A, Dawson PE: Expanding the scope of chemoselective peptide ligations in chemical biology. Curr. Opin. Chem. Biol. 12(6), 760-766 (2008).

176. Guerry P: Campylobacter flagella: not just for motility. Trends Microbiol. 15(10), 456-461 (2007).

177. Liu F, Aubry AJ, Schoenhofen IC, Logan SM, Tanner ME: The engineering of bacteria bearing azido-pseudaminic acid-modified flagella. Chembiochem 10(8), 1317-1320 (2009).

178. Saxon E, Bertozzi CR: Chemical and biological strategies for engineering cell surface glycosylation. Annu. Rev. Cell Dev. Biol. 17, 1-23 (2001).

179. Saxon E, Luchansky SJ, Hang HC, Yu C, Lee SC, Bertozzi CR: Investigating cellular metabolism of synthetic azidosugars with the Staudinger ligation. J. Am. Chem. Soc. 124(50), 14893-14902 (2002).

180. Pilobello KT, Krishnamoorthy L, Slawek D, Mahal LK: Development of a lectin microarray for the rapid analysis of protein glycopatterns. Chembiochem 6(6), 985-989 (2005).

181. Kuno A, Uchiyama N, Koseki-Kuno S et al: Evanescent-field fluorescence-assisted lectin microarray: a new strategy for glycan profiling. Nat. Methods 2(11), 851-856 (2005).
182. Ebe $\mathrm{Y}$, Kuno A, Uchiyama $\mathrm{N}$ et al.: Application of lectin microarray to crude samples: differential glycan profiling of lec mutants. J. Biochem. 139(3), 323-327 (2006).

183. Rudiger H, Gabius HJ: Plant lectins: occurrence, biochemistry, functions and applications. Glycoconj. J. 18(8), 589-613 (2001).

184. Cummings RD: Use of lectins in analysis of glycoconjugates. Methods Enzymol. 230, 66-86 (1994).

185. Hsu KL, Mahal LK: A lectin microarray approach for the rapid analysis of bacterial glycans. Nat. Protoc. 1(2), 543-549 (2006).

186. Hsu KL, Pilobello KT, Mahal LK: Analyzing the dynamic bacterial glycome with a lectin microarray approach. Nat. Chem. Biol. 2(3), 153-157 (2006).

187. Coyne MJ, Reinap B, Lee MM, Comstock LE: Human symbionts use a host-like pathway for surface fucosylation. Science 307(5716), 1778-1781 (2005).

188. Karlsson KA: The human gastric colonizer Helicobacter pylori: a challenge for hostparasite glycobiology. Glycobiology 10(8), 761-771 (2000).

189. Friis LM, Pin C, Pearson BM, Wells JM: In vitro cell culture methods for investigating Campylobacter invasion mechanisms. J. Microbiol. Methods 61(2), 145-160 (2005).

190. Lis H, Sharon N: Lectins: carbohydratespecific proteins that mediate cellular recognition. Chem. Rev. 98(2), 637-674 (1998).

191. Manimala JC, Li Z, Jain A, VedBrat S, Gildersleeve JC: Carbohydrate array analysis of anti-Tn antibodies and lectins reveals unexpected specificities: implications for diagnostic and vaccine development. Chembiochem 6(12), 2229-2241 (2005).

192. Morales BC, Gonzalez-Moa MJ, Boltz KW, Van der Werf BD, Johnston SA, Svarovsky SA: Bacterial glycoprofiling by using random sequence peptide microarrays. Chembiochem 10(5), 877-888 (2009).

193. Zhao C, Ge B, De Villena J et al:: Prevalence of Campylobacter spp., Escherichia coli, and Salmonella serovars in retail chicken, turkey, pork, and beef from the greater Washington, D.C., area. Appl. Environ. Microbiol. 67(12), 5431-5436 (2001).

194. Parkhill J, Wren BW, Mungall K et al.: The genome sequence of the food-borne pathogen Campylobacter jejuni reveals hypervariable sequences. Nature 403(6770), 665-668 (2000).

195. Whitfield C: Bacterial extracellular polysaccharides. Can. J. Microbiol. 34(4), 415-420 (1988)
196. Gilbert M, Mandrell RE, Parker CT, Li J, Vinogradov E: Structural analysis of the capsular polysaccharide from Campylobacter jejuni RM1221. Chembiochem 8(6), 625-631 (2007).

197. St Michael F, Szymanski CM, Li J et al.: The structures of the lipooligosaccharide and capsule polysaccharide of Campylobacter jejuni genome sequenced strain NCTC 11168. Eur. J. Biochem. 269(21), 5119-5136 (2002).

198. Ang CW, Noordzij PG, de Klerk MA, Endtz HP, van Doorn PA, Laman JD: Ganglioside mimicry of Campylobacter jejuni lipopolysaccharides determines antiganglioside specificity in rabbits. Infect. Immun. 70(9), 5081-5085 (2002).

199. Guerry P, Szymanski CM, Prendergast MM et al:: Phase variation of Campylobacter jejuni 81-176 lipooligosaccharide affects ganglioside mimicry and invasiveness in vitro. Infect. Immun. 70(2), 787-793 (2002).

200. Dzieciatkowska M, Brochu D, van BA et al. Mass spectrometric analysis of intact lipooligosaccharide: direct evidence for $O$-acetylated sialic acids and discovery of $O$-linked glycine expressed by Campylobacter jejuni. Biochemistry 46(50), 14704-14714 (2007).

201. Houliston RS, Endtz HP, Yuki N et al.: Identification of a sialate $O$-acetyltransferase from Campylobacter jejuni: demonstration of direct transfer to the C-9 position of terminala-2, 8-linked sialic acid. J. Biol. Chem. 281(17), 11480-11486 (2006).

202. Champion OL, Gaunt MW, Gundogdu O et al.: Comparative phylogenomics of the food-borne pathogen Campylobacter jejuni reveals genetic markers predictive of infection source. Proc. Natl Acad. Sci. USA $102(44)$, 16043-16048 (2005).

203. Thibault P, Logan SM, Kelly JF et al.: Identification of the carbohydrate moieties and glycosylation motifs in Campylobacter jejuni flagellin. J. Biol. Chem. 276(37), 34862-34870 (2001).

204. Logan SM, Kelly JF, Thibault P, Ewing CP, Guerry P: Structural heterogeneity of carbohydrate modifications affects serospecificity of Campylobacter flagellins. Mol. Microbiol. 46(2), 587-597 (2002).

205. Hendrixson DR, DiRita VJ: Identification of Campylobacter jejuni genes involved in commensal colonization of the chick gastrointestinal tract. Mol. Microbiol. 52(2), 471-484 (2004).

206. Joshua GW, Guthrie-Irons C, Karlyshev AV, Wren BW: Biofilm formation in Campylobacter jejuni. Microbiology 152(Pt 2), 387-396 (2006). 


\section{Review Reid, Fulton \& Twine}

207. Linton D, Allan E, Karlyshev AV, Cronshaw AD, Wren BW: Identification of $\mathrm{N}$-acetylgalactosamine-containing glycoproteins PEB3 and CgpA in Campylobacter jejuni. Mol. Microbiol. 43(2), 497-508 (2002).

208. Wyszynska A, Zycka J, Godlewska R, Jagusztyn-Krynicka EK: The Campylobacter jejunilcoli cjaA (cj0982c) gene encodes an $N$-glycosylated lipoprotein localized in the inner membrane. Curr. Microbiol. 57(3), 181-188 (2008).

209. Szymanski CM, Burr DH, Guerry P: Campylobacter protein glycosylation affects host cell interactions. Infect. Immun. 70 (4), 2242-2244 (2002).

210. Karlyshev AV, Everest P, Linton D, Cawthraw S, Newell DG, Wren BW: The Campylobacter jejuni general glycosylation system is important for attachment to human epithelial cells and in the colonization of chicks. Microbiology 150(Pt 6), 1957-1964 (2004).

211. Jones MA, Marston KL, Woodall CA et al.: Adaptation of Campylobacter jejuni NCTC11168 to high-level colonization of the avian gastrointestinal tract. Infect. Immun. 72(7), 3769-3776 (2004).

212. Helenius A, Aebi M: Intracellular functions of $N$-linked glycans. Science 291(5512), 2364-2369 (2001).

213. Fouts DE, Mongodin EF, Mandrell RE et al: Major structural differences and novel potential virulence mechanisms from the genomes of multiple Campylobacter species. PLoS Biol. 3(1), e15 (2005).
214. Guerry P, Szymanski CM: Campylobacter sugars sticking out. Trends Microbiol. 16(9), 428-435 (2008).

- Comprehensive review of current knowledge of the Campylobacter glycome and associated biosynthetic pathways.

215. Stintzi A, van Vliet AH, Ketley JM. Iron metabolism, transport, and regulation. In: Campylobacter. Nachamkin I, Szymanski CM, Blaser MJ (Eds). ASM Press, Washington, DC, USA, 591-610 (2008)

216. Blixt O, Razi N: Chemoenzymatic synthesis of glycan libraries. Methods Enzymol. 415, 137-153 (2006).

217. Sinclair AM, Elliott S: Glycoengineering: the effect of glycosylation on the properties of therapeutic proteins. J. Pharm. Sci. 94(8), 1626-1635 (2005).

218. Langdon RH, Cuccui J, Wren BW: $N$-linked glycosylation in bacteria: an unexpected application. Future Microbiol. 4(4), 401-412 (2009).

219. Faridmoayer A, Fentabil MA, Haurat MF et al:: Extreme substrate promiscuity of the Neisseria oligosaccharyl transferase involved in protein $O$-glycosylation. J. Biol. Chem. 283(50), 34596-34604 (2008).

\section{Website}

301. Mascot. www.matrixscience.com

\section{Affiliations}

- Christopher W Reid

National Research Council - Institute for Biological Science, Ottawa, Ontario, K1A 0R6, Canada

Tel.: +1 6139912141

Fax: +1 6139529092

chris.reid@nrc-cnrc.gc.ca

- Kelly M Fulton

National Research Council - Institute for Biological Science, Ottawa, Ontario, K1A 0R6, Canada

Tel.: +1 6139915466

Fax: +1 6139529092

kelly.fulton@nrc-cnrc.gc.ca

- Susan M Twine

National Research Council - Institute for Biological Science, Ottawa, Ontario, K1A 0R6, Canada

Tel.: +1 6139497545

Fax: +1 6139529092

susan.twine@nrc-cnrc.gc.ca 